\title{
Sign-Problem-Free Quantum Monte Carlo Study on Thermodynamic Properties and Magnetic Phase Transitions in Orbital-Active Itinerant Ferromagnets
}

\author{
Shenglong $\mathrm{Xu},{ }^{1} \mathrm{Yi} \mathrm{Li}^{2,{ }^{*}}$ and Congjun $\mathrm{Wu}^{1, \dagger}$ \\ ${ }^{1}$ Department of Physics, University of California, San Diego, California 92093, USA \\ ${ }^{2}$ Princeton Center for Theoretical Science, Princeton University, Princeton, New Jersey 08544, USA \\ (Received 21 December 2014; revised manuscript received 19 April 2015; published 23 June 2015)
}

\begin{abstract}
The microscopic mechanism of itinerant ferromagnetism is a long-standing problem due to the lack of nonperturbative methods to handle strong magnetic fluctuations of itinerant electrons. We nonpertubatively study thermodynamic properties and magnetic phase transitions of a two-dimensional multiorbital Hubbard model exhibiting ferromagnetic ground states. Quantum Monte Carlo simulations are employed, which are proved in a wide density region free of the sign problem usually suffered by simulations for fermions. Both Hund's coupling and electron itinerancy are essential for establishing the ferromagnetic coherence. No local magnetic moments exist in the system as a priori; nevertheless, the spin channel remains incoherent showing the Curie-Weiss-type spin magnetic susceptibility down to very low temperatures at which the charge channel is already coherent, exhibiting a weakly temperature-dependent compressibility. For the SU(2) invariant systems, the spin susceptibility further grows exponentially as approaching zero temperature in two dimensions. In the paramagnetic phase close to the Curie temperature, the momentum space Fermi distributions exhibit strong resemblance to those in the fully polarized state. The long-range ferromagnetic ordering appears when the symmetry is reduced to the Ising class, and the Curie temperature is accurately determined. These simulations provide helpful guidance to searching for novel ferromagnetic materials in both strongly correlated $d$-orbital transition-metal oxide layers and the $p$-orbital ultracold atom optical lattice systems.
\end{abstract}

DOI: 10.1103/PhysRevX.5.021032

\section{INTRODUCTION}

Itinerant ferromagnetism (FM) is one of the central topics of contemporary condensed-matter physics [1-23]. It has also become a research focus both experimentally and theoretically of ultracold atom physics [24-31]. The mechanism of itinerant FM has been a long-standing problem. Stoner proposed the exchange interaction among electrons with parallel spins as the driving force for itinerant FM [1]. Along this direction, the local-density approximation (LDA) of the density functional theory has achieved great success $[32,33]$. For example, the groundstate magnetic moments of FM metals can be calculated accurately [34]. The implementation of correlation effects in LDA has also been improved by the methods of LDA + $U$ [35], LDA + DMFT (dynamical mean-field theory) [36-38], and LDA + GP (Gutzwiller projection) [39-41].

\footnotetext{
${ }^{*}$ Corresponding author. y15@princeton.edu

Corresponding author. wucj@physics.ucsd.edu

Published by the American Physical Society under the terms of the Creative Commons Attribution 3.0 License. Further distribution of this work must maintain attribution to the author(s) and the published article's title, journal citation, and DOI.
}

Subject Areas: Atomic and Molecular Physics,

Magnetism, Strongly Correlated Materials
Nevertheless, itinerant FM systems are also strongly correlated, and their physics is typically nonperturbative. Usually repulsive interactions need to be sufficiently strong to overcome the kinetic energy cost of polarizing electron spins, and thus, itinerant FM has no well-controlled weakcoupling starting point. The Stoner criterion overlooks correlation effects among electrons with opposite spins [23]: Electrons can delicately organize their wave functions to reduce repulsions and still remain unpolarized even in the presence of strong interactions. For example, the LiebMattis theorem proves that the ground state of a rigorously 1D electron system with equal numbers of two spin components is a spin singlet no matter how strong the interaction [5].

It is more appropriate to start with electron orbitals to construct lattice model Hamiltonians to address the strong correlation aspect of itinerant FM. Exact theorems establishing FM, which are usually based on lattice models, are indispensable to provide reference points for further investigations. Well-known examples include the Nagaoka theorem [6,42-46], which applies to the infinite $U$ Hubbard models in two and above dimensions with doping a single hole on the half filled background, and the "flatband" FM in certain lattices with dispersionless band structures [13,47]. Recently, Nagaoka-type FM has also 
been generalized to 3D $t_{2 g}$-orbital systems in the cubic lattice with a quasi-2D band structure [48]. The Nagaokatype FM arises because the spin-polarized background maximally facilitates the hole's coherent hopping, while for the flatband case, the kinetic energy cost of polarizing spins is reduced to zero.

One central issue of itinerant FM is the role of orbital degeneracy, which widely exists in FM metals. Hund's coupling is a prominent feature in multiorbital systems, which favors electrons on the same site to align their spins. In some FM systems, itinerant electrons and local moments coexist and interact through Hund's coupling. When itinerant electrons are in the low-density limit, their motion can polarize the local moments through Hund's coupling [4], known as the double-exchange mechanism. However, Hund's coupling is local physics that usually cannot polarize itinerant electrons in the absence of local moments. Under what precise conditions Hund's coupling can lead to the global FM coherence in purely itinerant systems without local moments is still an open question.

The difficulty in achieving unambiguous FM ground states is only one side of the strong correlation physics of itinerant FM. The finite-temperature thermodynamic properties are another challenge [10,49-51]. At first sight, it might not look so striking: the FM susceptibilities show the standard mean-field Curie-Weiss (CW) law in the off-critical region as

$$
\chi(T)=\frac{C}{T-T_{0}},
$$

where $C$ is the Curie constant [52] and $T_{0}$ is the Curie temperature at the mean-field level. The $\mathrm{CW}$ law manifests spin incoherence, which is common in the paramagnetic state based on local moments. But it is difficult to understand why the CW law remains valid in itinerant FM metals possessing Fermi surfaces. For example, the transport and the charge channel properties, such as resistance and compressibility, are metallic; i.e., they are featured by the Fermi surface physics.

Within the itinerant picture, the Pauli magnetic susceptibility augmented by the RPA yields $\chi(T) \propto 1 /\left(T^{2}-T_{0}^{2}\right)$, but it is not commonly observed in experiments [10,50,51]. In fact, the CW law in FM metals applies to a wide range of temperatures $T_{f} \gg T>T_{0}$ ( $T_{f}$ is the Fermi temperature), which shows spin incoherence well below $T_{f}$. The reason is that RPA treats the paramagnetic phase as a weakly correlated Fermi liquid state with slightly thermally broadened Fermi distributions. Actually, this phase is rather complicated: Dynamic FM domains strongly fluctuate, which is beyond the RPA description and is difficult to handle analytically. The paramagnetic state of itinerant FM exhibits much higher entropy capacity than the usual weakly correlated paramagnetic Fermi liquid state, which significantly suppresses the genuine Curie temperature $T_{c}$, or the renormalized one, away from the mean-field value $T_{0}$. Consequently, $T_{c}$ is often significantly overestimated by weak-coupling approaches [10,50,51].

A key question is, how can itinerant systems exhibit the CW law and further develop FM purely based on itinerant electrons without involving local moments such that the charge channel remains coherent? Significant efforts have been made, including the self-consistent renormalization theory including spin-mode coupling $[9,10,49]$, the direct exchange from the Coulomb integral [11,53], spin incoherence due to Hund's coupling [54], and the orbital-selective Mott transition [55,56]. An important progress is that the $\mathrm{CW}$ law can be obtained from the combined method of LDA + DMFT [57] away from the critical region. However, none of these methods are nonperturbative in nature. and approximations are not well controlled.

Another issue is the nature of the FM phase transitions in FM metals, which has been widely studied but is still under intensive debate $[8,14,16-19]$. Compared to the superconducting phase transitions in which the fermion degree of freedom is gapped below transition temperatures, the FM phase transitions are more involved because systems remain gapless across transitions due to the existence of Fermi surfaces. The FM domain fluctuations combined with the Landau damping from the gapless particle-hole excitations around Fermi surfaces complicates the FM phase transitions. Because of the strong correlation nature of the problem, a well-controlled nonperturbative study would be desired and it remains a big challenge.

Recently, the ground states of a multiorbital Hubbard model have been proved fully spin polarized in the strongcoupling regime in the 2D square and 3D cubic lattices [21] by two of us and Lieb. It it shown that interorbital Hund's coupling combined with electron itinerancy in the quasi-1D band structure drives the FM ground states. Compared to the Nagaoka FM, this new theorem proves a stable FM phase with nodeless ground-state wave functions over the entire electron density region $0<n<2$, where $n$ is the occupation number per site; thus, it sets up a solid starting point for further studying the strong correlation aspect of itinerant FM. It also opens up the possibility of performing sign-problem-free quantum Monte Carlo (QMC) simulations away from half filling by employing the bases under which the many-body Hamiltonians satisfy the Perron-Frobenius condition.

Although this theorem sets up only the ground-state properties, it establishes an unambiguous FM phase as a starting point for further studying both thermodynamic properties and magnetic phase transitions over a wide region of electron density. In order to handle the strong magnetic fluctuations, QMC simulations would be the ideal method; however, they usually suffer the notorious sign problem for fermions and thus are, generally speaking, inapplicable for itinerant FM. Remarkably, we prove that for the systems in which the ground-state FM theorem 
mentioned above [21] applies, the fermion sign problem can be eliminated in the entire electron density region. This provides a new opportunity to study the finite-temperature thermodynamic properties and magnetic phase transitions in an asymptotically exact way.

In this article, we present a systematic nonperturbative study on thermodynamic properties and magnetic phase transitions of itinerant FM by performing sign-problemfree QMC simulations. Our results show that itinerant FM can indeed exhibit both spin incoherence and charge coherence simultaneously without forming local moments. In other words, the system exhibits the feature of the CW metal as a combined effect of Hund's coupling and electron itinerancy. The model we simulate can be realized in both $d$-orbital transition-metal oxide layer and $p$-orbital ultracold atom optical lattices, which do not contain local moments as a priori. The spin magnetic susceptibility exhibits the CW law as a signature of spin incoherence, while the compressibility weakly depends on temperature as a consequence of itinerancy. The mean-field Curie temperature $T_{0}$ is extracted based on the $\mathrm{CW}$ law in the off-critical region, which is much lower than the temperature scale of charge coherence $T_{\mathrm{ch}}$. The filling dependence of $T_{0}$ is calculated and the maximal $T_{0}$ reaches one-tenth of the hopping integral. The Fermi distribution functions in momentum space are calculated in the strongly correlated paramagnetic phase. The fermion occupation numbers are strongly suppressed from the saturated value even for wave vectors close to the center of the Brillouin zone. When entering the critical region, for the $\mathrm{SU}(2)$ symmetric models, $\chi(T)$ grows exponentially. The true FM long-range order is achieved by reducing the model symmetry to the Ising class, and the FM critical temperature $T_{c}$ is determined accurately by the finite size and critical scalings.

For later convenience, we briefly introduce the FM critical fluctuations here, which are particularly important in two dimensions. According to the Landau-GinzburgWilson paradigm of critical phenomena, $T_{c}$ is suppressed from $T_{0}$ but remains finite in $3 \mathrm{D}$. As $T$ is lowered from $T_{0}$ and approaches $T_{c}$, the system crosses over from the meanfield region to the critical region, and $\chi(T) \propto\left(T-T_{c}\right)^{-\gamma}$ due to non-Gaussian fluctuations and $\gamma$ is the critical exponent. In 2D, $T_{c}$ remains finite if the system symmetry is reduced to the Ising class, or the easy axis class. However, for the isotropic class, thermal fluctuations suppress $T_{c}$ to zero according to the Mermin-Wagner theorem [58,59]. Nevertheless, even in this case, the mean-field $T_{0}$ is still an important temperature scale below which the FM order develops its magnitude. However, the orientation fluctuations of the FM order suppress the long-rang order. In other words, this region is characterized by fluctuating FM domains and the correlation length increases exponentially as lowering temperatures. Consequently, the FM susceptibility deviates from the CW law and crosses over into an exponential growth.
The rest of this article is organized as follows. In Sec. II, the model Hamiltonian is introduced, and the QMC method for this model is proved free of the sign problem. The QMC simulations on the thermodynamic properties in the offcritical region is presented in Sec. III, and the momentum space Fermi distributions are calculated in Sec. IV. The results in the critical region are presented in Sec. V. In Sec. VI, we discuss the physics when the conditions for the absence of the sign problem are loosened. Simulations in the presence of a small interchain hopping, in which the sign problem appears but is not severe, are presented. The experimental realizations are discussed in Sec. VII. Conclusions are made in Sec. VIII.

\section{MODEL HAMILTONIAN AND THE ABSENCE OF THE SIGN PROBLEM}

In this section, we present the model Hamiltonians, whose ground states were proved to be ferromagnetic [21]. Furthermore, we also explain that the QMC sign problem is absent, and thus, this model provides an ideal preliminary to study the thermodynamic properties and magnetic phase transitions of strongly correlated itinerant FM in a controllable way.

\section{A. Model Hamiltonians}

We consider the case of the 2D square lattice: On each site there are two orthogonal orbitals forming a quasi-1D band structure. For simplicity, below we use the 2D $p$-orbital system as an example, and the physics is also valid for the $d_{x z^{-}}$and $d_{y z}$-orbitals systems in 2D. The relevance of this model to the current experiment efforts of searching for novel itinerant FM systems is discussed in Sec. VI. For the band structure, we keep only the $\sigma$-bonding $t_{\|}$term; i.e., electrons in the $p_{x(y)}$ orbital move only longitudinally along the $x(y)$ direction, respectively. The following Hamiltonian is defined in the square lattice as

$$
\begin{aligned}
H_{\text {kin }, \|}= & -t_{\|} \sum_{\vec{r}, \sigma}\left\{p_{x \sigma}^{\dagger}\left(\vec{r}+\hat{e}_{x}\right) p_{x \sigma}(\vec{r})+p_{y \sigma}^{\dagger}\left(\vec{r}+\hat{e}_{y}\right)\right. \\
& \left.\times p_{y \sigma}(\vec{r})+\text { H.c. }\right\}-\mu \sum_{\vec{r}} n(\vec{r}),
\end{aligned}
$$

in which we neglect the small transverse bonding $t_{\perp}$ term. For realistic $p$-orbital systems, the sign of $t_{\|}$is negative due to the odd parity of $p$-orbital Wannier wave functions. Nevertheless, for the bipartite lattice, such as the square lattice, the sign of $t_{\|}$can be flipped by a gauge transformation. Without loss of generality, $t_{\|}$is scaled to 1 below, which serves as the unit for all other quantities carrying energy unit in this article.

The interaction part $H_{\text {int }}$ contains the standard multiorbital Hubbard interaction [60-63] as 


$$
\begin{aligned}
H_{\mathrm{int}}= & U \sum_{\vec{r}, a=x, y} n_{a, \uparrow}(\vec{r}) n_{a, \downarrow}(\vec{r})+V \sum_{\vec{r}} n_{x}(\vec{r}) n_{y}(\vec{r}) \\
& -J \sum_{\vec{r}}\left\{\vec{S}_{x}(\vec{r}) \cdot \vec{S}_{y}(\vec{r})-\frac{1}{4} n_{x}(\vec{r}) n_{y}(\vec{r})\right\} \\
& +\Delta \sum_{\vec{r}}\left\{p_{x \uparrow}^{\dagger}(\vec{r}) p_{x \downarrow}^{\dagger}(\vec{r}) p_{y \downarrow}(\vec{r}) p_{y \uparrow}(\vec{r})+\text { H.c. }\right\},
\end{aligned}
$$

where $a=x, y$ refer to the orbital index, $n_{a, \sigma}=p_{a, \sigma}^{\dagger} p_{a, \sigma}$ and $n_{a}=n_{a, \uparrow}+n_{a, \downarrow}$, and $\vec{S}_{a}=p_{a, \alpha}^{\dagger} \vec{\sigma}_{\alpha \beta} p_{a, \beta}$ is the spin operator of the $a$ orbital. The $U$ and $V$ terms describe the intraorbital and interorbital Hubbard interactions, respectively, the $J$ term is Hund's coupling and $J>0$ represents its FM nature, and the $\Delta$ term describes the pairing hopping process between two orthogonal orbitals.

In order to gain an intuitive understanding of the interaction parameters, we consider a single site problem. There are in total six states that can be classified as a set of spin-triplet states and three different spin-singlet states. The triplet states are with energy $V$, defined as

$p_{x, \uparrow}^{\dagger} p_{y, \uparrow}^{\dagger}|0\rangle, \quad \frac{1}{\sqrt{2}}\left(p_{x, \uparrow}^{\dagger} p_{y, \downarrow}^{\dagger}+p_{x, \downarrow}^{\dagger} p_{y, \uparrow}^{\dagger}\right)|0\rangle$,

$p_{x, \downarrow}^{\dagger} p_{y, \downarrow}^{\dagger}|0\rangle$,

respectively, where $|0\rangle$ is the vacuum state. The other three spin-singlet states are

$\frac{1}{\sqrt{2}}\left(p_{x, \uparrow}^{\dagger} p_{y, \downarrow}^{\dagger}-p_{x, \downarrow}^{\dagger} p_{y, \uparrow}^{\dagger}\right)|0\rangle, \quad p_{x, \uparrow}^{\dagger} p_{x, \downarrow}^{\dagger}|0\rangle$,

$p_{y, \uparrow}^{\dagger} p_{y, \downarrow}^{\dagger}|0\rangle$,

among which the first one involves both orbitals and its energy is $V+J$; the other two singlets only occupy the same orbital with the average energy $U$ and the hybridization matrix element between them is $\Delta$. In the limit of $U \rightarrow+\infty$, the states of $p_{x, \uparrow}^{\dagger} p_{x, \downarrow}^{\dagger}|0\rangle$ and $p_{y, \uparrow}^{\dagger} p_{y, \downarrow}^{\dagger}|0\rangle$ are projected out. Nevertheless, the other four doubly occupied states are kept in the physical Fock space, including one set of spin-triplet states and one interorbital singlet state.

The ground states of the Hamiltonians Eq. (2) plus Eq. (3) are fully spin polarized at any generic filling $n$ for arbitrary values of $V$ under the condition that $U \rightarrow+\infty$ and $J>0$. The detailed proof and its generalization to the 3D cubic lattice are presented in Ref. [21]. Below, we present an intuitive physical picture. The band structure of Eq. (2) is quasi-1D, consisting of orthogonal rows and columns, and electrons do not transit among different lines. In the absence of Hund's coupling, the intrachain physics in the limit of $U \rightarrow+\infty$ would then correspond to the 1D infinite$U$ Hubbard model whose ground states are highly degenerate regardless of the spin configurations. Now, let us turn on $J>0$, and the interchain Hund's coupling lifts the degeneracy and selects the fully polarized state as the unique ground state: When one electron in a row meets another one in a column at the crossing site, their spins are aligned to save the energy of $J$, which is, thus, different from the usual case that Hund's coupling can only polarize electrons on the same site. Remarkably, in this case, it does polarize electrons in the entire system [20,21]. Although the electron band structure is quasi-1D, interactions couple electron spins in different chains together, and thus, the FM correlations and ordering are genuinely $2 \mathrm{D}$ or $3 \mathrm{D}$.

For completeness, we also present the Hamiltonian of the interchain hopping with a small value of $t_{\perp}$ as

$$
\begin{aligned}
H_{\mathrm{kin}, \perp}= & -t_{\perp} \sum_{\vec{r}, \sigma}\left\{p_{x \sigma}^{\dagger}\left(\vec{r}+\hat{e}_{y}\right) p_{x \sigma}(\vec{r})\right. \\
& \left.+p_{y \sigma}^{\dagger}\left(\vec{r}+\hat{e}_{x}\right) p_{y \sigma}(\vec{r})+\text { H.c. }\right\},
\end{aligned}
$$

which is used in Sec. VI A. Again, in the square lattice the sign of $t_{\perp}$ can be flipped by a gauge transformation, and without loss of generality, it is assumed to be positive. We set $t_{\perp}=0$ in most part of this article except in Sec. VI A.

\section{B. Absence of the QMC sign problem}

The many-body Hamiltonian matrix of Eq. (2) plus Eq. (3) possesses an important sign structure in the limit of $U \rightarrow+\infty$ under which the ground-state FM theorem applies [21]. In the coordinate representation, a convenient set of many-body bases are defined by ordering fermions according to their real-space positions along one row by another and then along one column by another. The periodical and antiperiodical boundary conditions are employed for each chain if the particle number in that chain is odd or even, respectively, which is feasible because the particle number in each chain is separately conserved. This particular choice of boundary conditions should not change the bulk physics. Under these bases and boundary conditions, in the limit of $U \rightarrow+\infty$, the electron hopping term and the spin-flip term from Hund's coupling do not change the sequence of fermion ordering. When electrons hop across the boundary, no extra minus sign appears either, due to the above boundary condition. Then the many-body Hamiltonian matrix satisfies the prerequisite of the Perron-Frobenius theorem: All the nonzero off-diagonal matrix elements are either $-t$ or $-J$ arising from the kinetic energy term and Hund's coupling, respectively, and thus, they are semi-negative-definite. We do not need to consider the pair hopping process, which is completely suppressed in the limit of $U \rightarrow+\infty$. Remarkably, the above sign structure of the off-diagonal matrix elements renders the ground-state many-body wave function nodeless, and also leads to the disappearance of the QMC sign problem for the ground states.

For the finite-temperature thermodynamic properties, we use the stochastic series expansion (SSE) QMC method with the directed loop update algorithm [64-68]. This method is usually used for boson systems and 1D fermion 
systems. In our case, although the band structure of Eq. (2) is quasi-1D-like, the interaction Eq. (3) couples all the chains together. In particular, the total spin of each chain is not conserved, and thus, its magnetic properties is truly two dimensional. Remarkably, we find for this model that the sign problem is absent at finite temperatures in the entire electron density region $0<n<2$. In the SSE method, the partition function is expanded as

$$
Z=\sum_{n=0}^{+\infty} \frac{\beta^{n}}{n !} \sum_{\alpha_{i}^{n}} \prod_{i=1}^{n}\left\langle\alpha_{i}^{n}|-H| \alpha_{i-1}^{n}\right\rangle,
$$

where $H=H_{\text {kin, }}+H_{\text {int }} ;\left|\alpha_{i}^{n}\right\rangle$ runs over the set of manybody bases defined above and $\left|\alpha_{n}^{n}\right\rangle=\left|\alpha_{0}^{n}\right\rangle$. A negative constant is added to the many-body Hamiltonian matrix to make all of its diagonal matrix elements negative, and then all the matrix elements of $-H$ become positive. The grand canonical ensemble is employed to ensure the ergodicity of the particle number distribution in each chain. The parameters for the QMC simulations are provided in Appendix A.

The QMC sign problem does appear in the presence of the $t_{\perp}$ term, i.e., Eq. (6), because electrons become mobile in a two-dimensional manner. Nevertheless, the QMC simulations can still be performed when the sign problem is not so severe, which we present in Sec. VI A.

\section{THERMODYNAMIC QUANTITIES IN THE OFF-CRITICAL REGION}

In this section, we present the results of QMC simulations on the spin magnetic susceptibility $\chi(T)$ and the compressibility $\kappa(T)$ in the off-critical temperature region. $\chi(T)$ exhibits the celebrated $\mathrm{CW}$ law at temperatures well below the kinetic energy scale of the system, while $\kappa(T)$ typically weakly depends on temperature.

\section{A. Temperature dependence of spin susceptibility $\chi(T)$ and compressibility $\boldsymbol{\kappa}(\boldsymbol{T})$}

The spin susceptibility $\chi$ and compressibility $\kappa$ are two fundamental thermodynamic properties in interacting fermion systems in the spin and charge channels, respectively. In usual paramagnetic Fermi liquid states, both $\chi$ and $\kappa$ at zero temperature exhibit the itinerant feature controlled by the density of states at the Fermi energy. Furthermore, they are renormalized by interaction effects characterized by the Landau parameters $F_{a}^{0}$ and $F_{s}^{0}$ in the spin and charge channels, respectively. At finite temperatures much lower than the Fermi temperature, $\chi(T)$ and $\kappa(T)$ are only weakly temperature dependent. However, in FM metals, $\chi(T)$ and $\kappa(T)$ behave dramatically differently exhibiting localmoment-like and itinerant features, respectively, which we show from the QMC simulation results.

Because the total spin is conserved, the spin magnetic susceptibility $\chi$ is represented by the equal-time correlation function as

$$
\chi(T)=\lim _{L \rightarrow+\infty} \frac{\beta}{L^{2}} \sum_{\vec{r}_{1}, \vec{r}_{2}}\left\langle S_{z}\left(\vec{r}_{1}\right) S_{z}\left(\vec{r}_{2}\right)\right\rangle
$$

The QMC results of $\chi^{-1}(T)$ at $V=0$ are presented in Fig. 1(a) in the off-critical region based on the finite-size scalings shown in Appendix B. For all the values of $n$ presented, $\chi$ exhibits the $\mathrm{CW}$ law in the off-critical region. The values of $T_{0}$ extracted from the linear form $\chi^{-1}(T)$ range from 0.01 to 0.1 , which means that spin remains incoherent at temperatures well below $t_{\|}$(scaled to 1).

It is not surprising that $\chi(T)$ should asymptotically scale as $1 / T$ in the high-temperature limit $T \gg T_{f}$, where $T_{f}$ is the Fermi temperature, because in this limit, the spin channel is completely incoherent. Nevertheless, the spin incoherence persists into a much lower temperature scale $T_{0}$ below $T_{f}$. Although $T_{0}$ is a mean-field energy scale and is not the actual temperature for the onset of long-range order, it remains an important quantity. Physically, it
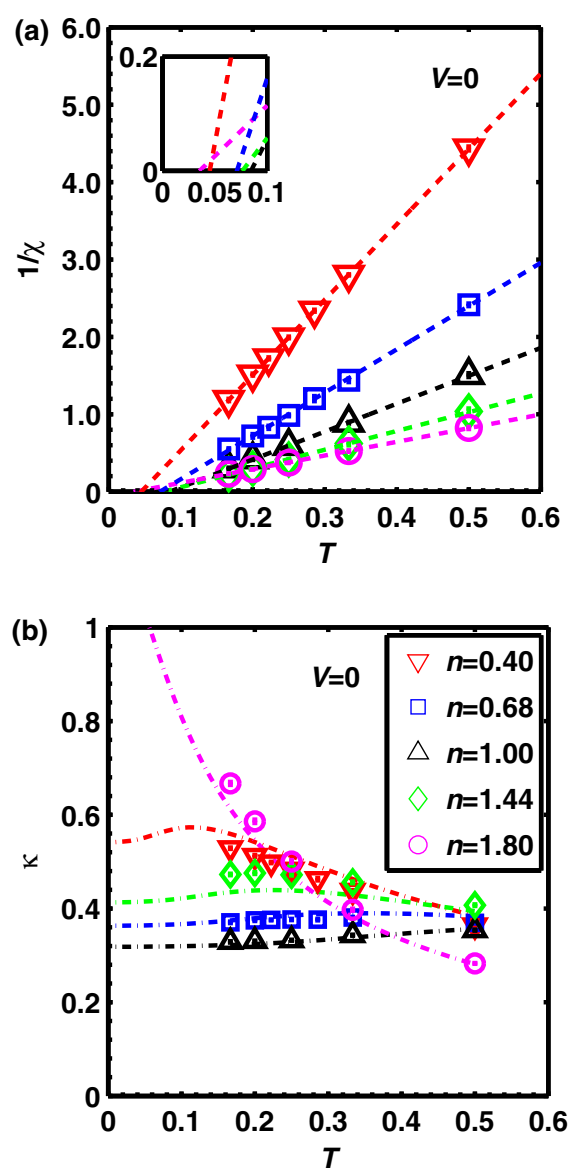

FIG. 1. (a) $\chi^{-1}(T)$ exhibits the CW law at different values of $n$. The inset shows interceptions corresponding to the mean-field value of Curie temperature $T_{0}$. (b) The compressibility $\kappa(T)$ at different values of $n$. The dashed lines represent $\kappa(T)$ of $1 \mathrm{D}$ spinless fermions at the same densities for comparison. Values of $n$ in (a) and (b) are represented by the same legend. $V=0$ and $J=2$ for both figures. The error bars of the QMC data are smaller than the symbols. 
roughly equals the energy cost of flipping an individual electron spin in the ground state. Because of non-Gaussian fluctuations, the actual FM critical temperature $T_{c}$ significantly deviates from $T_{0}$ defined in Eq. (1). In the current $\mathrm{SU}(2)$ invariant case, actually $T_{c}=0$, due to the MerminWagner theorem [58].

The compressibility $\kappa(T)$ reflects the coherence in the charge channel. Because the total particle number is a conserved quantity, it is also defined as an equal-time correlation function as

$$
\kappa(T)=\lim _{L \rightarrow+\infty} \frac{\beta}{L^{2}} \sum_{\vec{r}_{1}, \vec{r}_{2}}\left\langle n\left(\vec{r}_{1}\right) n\left(\vec{r}_{2}\right)\right\rangle
$$

The QMC results of $\kappa(T)$ at $V=0$ are presented in Fig. 1(b). Again, $\kappa$ is proportional to $1 / T$ in the hightemperature incoherent regime, as shown in Eq. (9), and it saturates at low temperatures in the metallic phase. The crossover temperature scale $T_{\mathrm{ch}}$ between these two regimes is typically the chemical potential at zero temperature. In the usual Fermi liquid state, $\kappa$ is typically the density of state at the Fermi energy renormalized by Landau parameters. In our case, the situation is different due to the prominent FM fluctuations. At $V=0$, due to the infinite $U$ and the 1D band structure, $T_{\mathrm{ch}}$ is roughly the Fermi temperature of spinless fermions at the same density. For most of the values of $n$ presented in Fig. 1(b), $T_{\text {ch }}$ is at the order of $t_{\|}$, and thus, $\kappa$ saturates in the temperature region presented. As for the case of a low hole density $n=1.8, T_{\text {ch }}$ can be estimated around 0.1 , and thus, $\kappa(T)$ does not saturate yet in the simulated temperature region. Because of the strong FM tendency, the interorbital interaction vanishes at $V=0$, and $\kappa(T)$ can be well fitted by that of spinless fermions, as shown in Fig. 1(b).

Comparing $\chi(T)$ and $\kappa(T)$, the spin coherence temperature $T_{0}$ is much lower than the charge coherence temperature $T_{\mathrm{ch}}$. These two distinct coherence temperature scales in spin and charge channels are an important feature of FM metals. A common phenomenological interpretation is to divide electrons into two parts: local moments and itinerant electrons, which are responsible for the spin and charge channel behaviors, respectively. However, this dividing is artificial for metals when all the electrons are itinerant with equivalent band structures such as in our case. Based on the QMC simulations above, we show unambiguously that the CW law can indeed appear in strongly correlated systems without local moment formation. A similar feature also appears in the CW metal states $[69,70]$ and the $1 \mathrm{D}$ spin incoherent Luttinger liquids [71]. The difference is the behavior of $\chi$ below the spin coherence temperature $T_{0}$. In the case of the CW metal, $\chi$ saturates, exhibiting the Paulilike behavior but strongly enhanced by interactions, and in the 1D case, antiferromagnetic correlations develops. In our case, as we show in Fig. 6, $\chi$ evolves into an exponential growth as a reminiscence of the FM long-range-ordered ground state [21].
Next, we consider the effects of a large interorbital repulsion $V$ to $\chi^{-1}(T)$ and $\kappa(T)$. The ground states remain fully spin polarized as proved in Ref. [21], and the QMC results of $\chi^{-1}(T)$ still exhibit the $\mathrm{CW}$ law at all the fillings, as shown in Fig. 2(a). The most prominent effect of $V$ is the suppression of $\kappa(T)$ at the commensurate filling of $n=1$, as shown in Fig. 2(b), in which the system is in the Mottinsulating state. In this case, electrons become local moments due to the opening of the charge gap. As a result, $\kappa(T)$ is suppressed to nearly zero at $0<T<0.5$, which is still small compared to the charge gap at the order of $V$. In the Mott-insulating ground state at $n=1$, the orbital channel can develop antiferro-orbital ordering with a staggered occupation of $p_{x}$ and $p_{y}$ orbitals. The QMC simulation results on the antiferro-orbital ordering transition are presented in Appendix C. As $n$ moves away from 1 , electrons become itinerant again. Nevertheless, the values of $\kappa(T)$ at $V=8$ are significantly suppressed compared to those with the same values of $n$ and $T$ at $V=0$.
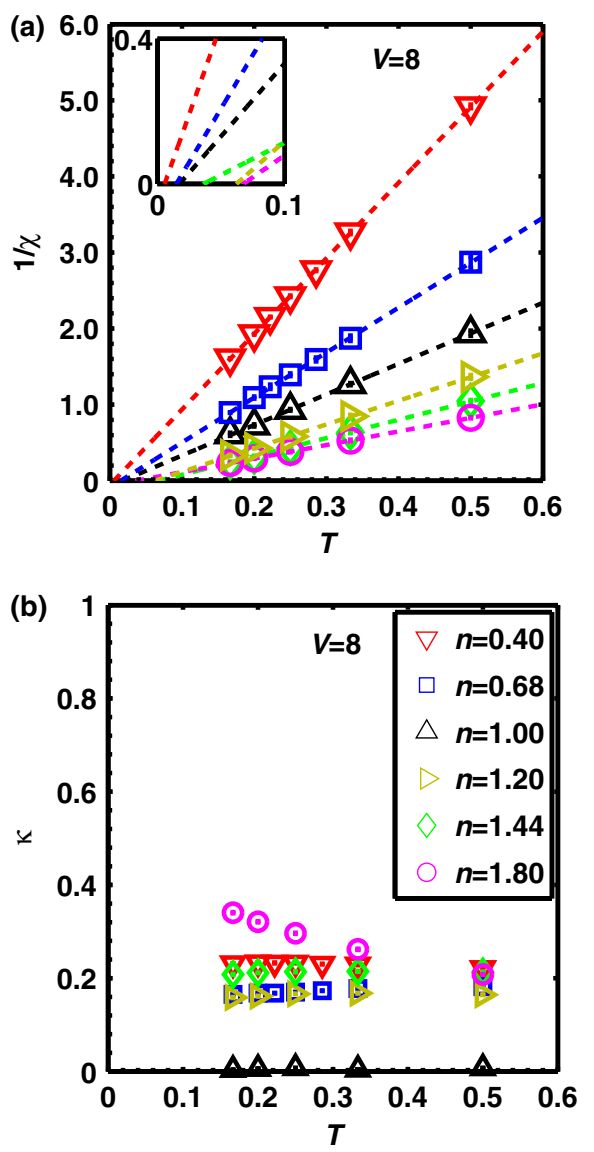

FIG. 2. (a) $\chi^{-1}(T)$ and (b) $\kappa(T)$ at a large value of $V=8$ in the temperature regime of $1 / 6<T<1 / 2$. Different values of $n$ are shown in the legend in (b). At $n=1$, the system is in the Mottinsulating state, and thus $\kappa(T)$ drops to nearly zero at low temperatures. The error bars of the QMC data are smaller than the symbols. 


\section{B. Density dependences of $T_{0}(n)$ and the Curie constant $\boldsymbol{C}(\boldsymbol{n})$}

The ground-state FM survives in all the filling region $0<n<2$, nevertheless, its robustness against thermal fluctuations varies at different densities, which reflects through the density dependences of $T_{0}(n)$ and $C(n)$.

The relation $T_{0}(n)$ is presented in Fig. 3(a) for both cases of $V=0$ and $V=8$. The FM coherence is built up due to the itinerancy of fermions [21]; thus, $T_{0}$ approaches zero in both limits of $n \rightarrow 0$ (the particle vacuum) and $n \rightarrow 2$ (the hole vacuum). At $V=0$, the maximal $T_{0}$ appears around $n=1$, where electrons are most mobile. $T_{0}(n)$ at $V=0$ is nearly symmetric with respect to $n=1$, exhibiting an approximate particle-hole symmetry. In contrast, it is highly asymmetric at large $V$. In this case, $T_{0}$ is strongly suppressed at $0<n<1$, in which both charge and spin carriers are electrons. A large $V$ penalizes two electrons occupying the same site; thus, the effectiveness of Hund's rule is suppressed. After $n$ passes 1, a quick increase of $T_{0}$ appears because extra electrons on top of the Mott background of $n=1$ can move easily to build up the FM
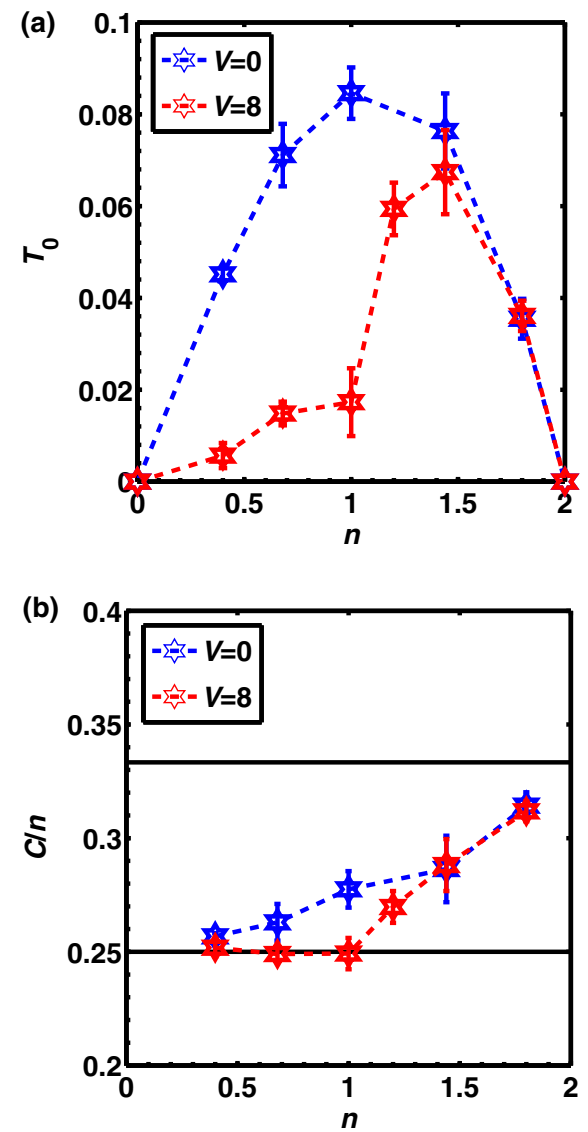

FIG. 3. (a) The density dependence of the Curie temperature $T_{0}(n)$ at $V=0$ and 8 with $J=2$, respectively. (b) The density dependence of the reduced Curie constant: $C / n$ versus $n$. The lower and upper bold lines represent the limits of the spin- $\frac{1}{2}$ and spin-1 moments, respectively. Plots are based on the results of $\chi(T)$ in Figs. 1(a) and 2(a). coherence. $T_{0}$ reaches the maximum roughly at the middle point between $n=1$ and 2. As $n \rightarrow 2, T_{0}$ becomes insensitive to $V$. In this region, most sites are doubly occupied in the states of spin-1 moments, and holes are itinerant but do not carry spin. The hole's motion threads spin moments along its trajectory and aligns their orientations, and this process is not much affected by $V$. At $V=8 t_{\|}$and $J=2 t_{\|}$, the maximal $T_{0} \approx 0.06 t_{\|}$, which appears around $n \approx 1$.4. In other words, at large values of $V$, there is an approximate particle-hole symmetry between $n=1-2$ on the background of one electron per site.

Now, we present the results of the Curie constant $C$. Assuming the local moment picture, the simple molecule field method yields $C$ per spin moment as $\frac{1}{3} S(S+1)$ [10], where $S$ is the spin magnitude. In our case mostly itinerant, the magnitudes of $S$ fluctuate: $C=0$ for the empty site, $\frac{1}{4}$ for the singly occupied site, and $\frac{2}{3}$ for the doubly occupied site in the spin-1 configuration, respectively. We plot the normalized Curie constant $C / n$ versus $n$ in Fig. 3(b). $C / n$ approaches $\frac{1}{4}$ as $n \rightarrow 0$, and $\frac{1}{3}$ as $n \rightarrow 2$, where most sites are spin-1 moments. Generally, $C / n$ lies between these two limits. At $V=0$, as $n$ increases, the number of on-site triplets smoothly increases and so does $C / n$. Nevertheless, at large $V$, the on-site triplet formation is strongly suppressed at $0<n<1$, and thus $C / n$ is stuck at $\frac{1}{4}$. After $n$ passes $1, C / n$ starts to increase nearly linearly toward $1 / 3$. As $n \rightarrow 2, V$ hardly affects the number of on-site triplets, and thus, $C / n$ also becomes insensitive to $V$ as $T_{0}$ does.

\section{On-site charge fluctuations and spin moments}

To further clarify the nature of our system, whether it is itinerant or local-moment-like, we calculate the on-site charge fluctuations and the average spin moments below. The on-site charge fluctuations are defined as

$$
\delta=\left\langle n_{i}^{2}\right\rangle-n^{2},
$$

where $n_{i}$ is the total particle number on site $i$. Because of the translation symmetry, $\delta$ is independent of the site index $i$, and the simulation results are plotted in Fig. 4. At $V=0$, the charge fluctuations are significant in the entire filling region except very close to the particle vacuum at $n=0$ and the hole vacuum at $n=2$. The maximum is reached at the approximate particle-hole symmetric point of $n=1$. The large on-site charge fluctuations clearly reflect the itinerant nature of the system, which is consistent with the compressibility results in Fig. 1(b). When the interorbital repulsion $V$ goes large, charge fluctuations are greatly suppressed near the commensurate filling $n=1$. In this case, the system becomes local-moment-like, which agrees with the vanishing compressibility shown in Fig. 2(b). Nevertheless, as moving away from $n=1$, the system becomes itinerant again exhibiting significant on-site charge fluctuations.

We also calculate the square of the $z$ component of the on-site spin moment $\left\langle S_{i, z}^{2}\right\rangle$, which equals $\frac{1}{3}\left\langle\vec{S}_{i}^{2}\right\rangle$, 


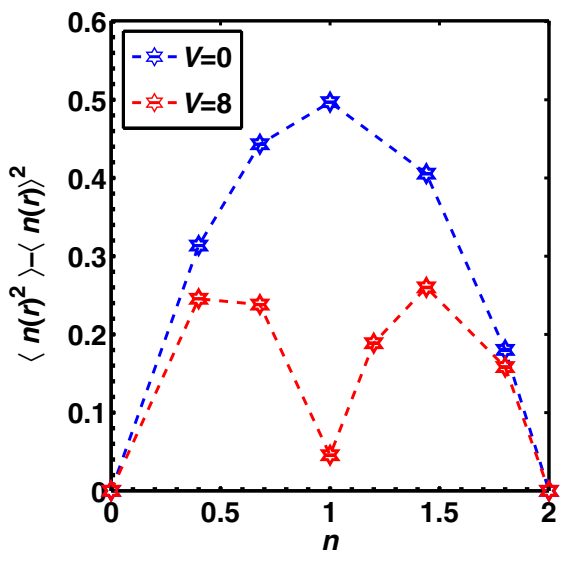

FIG. 4. The on-site particle number fluctuation $\delta$ defined in Eq. (10). The parameters are $\beta=6, J=2$, and $L=30$.

since the SU(2) symmetry is not broken. In order to compare with the Curie constant $C / n$, we plot its values normalized by the filling, i.e., $\left\langle S_{i, z}^{2}\right\rangle / n$ as presented in Fig. 14, which is nearly the same as the Curie constant $C / n$ plotted in Fig. 3(b). At $V=0,\left\langle\vec{S}_{i}^{2}\right\rangle$ varies smoothly with $n$ : the probable on-site configurations include empty, singly occupied (spin- $-\frac{1}{2}$ ), and doubly occupied (spin-1) states. At $V=8$ and the commensurate filling $n=1,\left\langle\vec{S}_{i}^{2}\right\rangle \approx \frac{3}{4}$, which manifests the formation of the local moment of spin $-\frac{1}{2}$, which is consistent with the suppressed charge fluctuations. At $0<n<1$, each site is nearly either empty or singly occupied, and thus, $\left\langle\vec{S}_{i}^{2}\right\rangle \approx \frac{3}{4} n$. At $1<n<2$, the probable on-site configurations include the singly occupied spin- $\frac{1}{2}$ moment and doubly occupied spin-1 moment. The nature of the variable valence of these states clearly shows the itinerancy of the system even at large $V$ when moving away from $n=1$.

\section{MOMENTUM SPACE FERMION OCCUPATION}

An important feature of the itinerant FM is the fluctuating FM domains in real space in the paramagnetic phase close to $T_{0}$. This prominent FM fluctuation also strongly affects the momentum space fermion occupation as shown below. Basically, the fermion occupation functions still resemble those in the fully polarized systems with thermal broadening.

Because the particle number of each chain is separately conserved, the momentum space distribution function is essentially 1D-like. Nevertheless, each chain is not isolated but interacts with others through multiorbital interactions, and thus, spin is not conserved separately in each chain. Without loss of generality, we define $n_{F}(k)=$ $\sum_{\sigma}\left\langle p_{x, \sigma}^{\dagger}(k) p_{x, \sigma}(k)\right\rangle$ for a horizontal $x$ chain. The case of $n=1$ is studied below as a representative, which is equivalent to $n_{x}=0.5$ in this $x$ chain. Its mean-field Curie temperature $T_{0} \approx 0.08$, as shown in Fig. 3(a). The

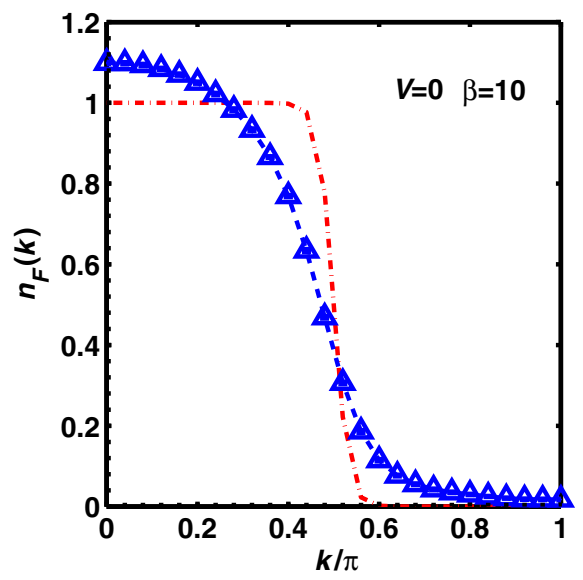

FIG. 5. The momentum space distribution $n_{F}(k)(0 \leq k \leq \pi)$ at $\beta=10$ and $n=1$, and $n_{F}(k)$ of the noninteracting spinless fermion (red dashed line) is plotted for comparison. The system size $L=50$. The parameter values are $V=0$ and $J=2$. The error bars of the QMC data are smaller than the symbol.

simulated results of $n_{F}(k)$ are presented in Fig. 5 with the periodical boundary condition, and a discussion on the boundary condition is presented in Appendix B.

We define a reference wave vector as the Fermi wave vector $k_{f}^{0}=\pi / 2$ of spinless fermions at the same density. At a low temperature $T=1 / \beta=0.1$ close to $T_{0}$, as shown in Fig. $5, n_{F}(k)$ is only slightly larger than 1 even at $k \ll k_{f}^{0}$. It smoothly decays to zero with a half-width approximately equal to $k_{f}^{0} \cdot n_{F}(k)$ is rounded off compared to that of spinless fermions at the same temperature. Although $n_{F}(k)$ does not look much different from that of spinless fermions, it is a consequence of strong interactions because the system is in the paramagnetic state. The system remains unpolarized with a FM correlation length $\xi$ at the order of 10-20, as estimated in Appendix B, and the upper bound of $n_{F}(k)=2$ as $k \rightarrow 0$.

The above result implies that the phase space for thermal fluctuations is not restricted to a small region close to $\pm k_{f}^{0}$, and thus, its entropy capacity is enhanced. It is consistent with the real-space picture of fluctuating FM domains as $T$ approaches $T_{0}$. This is highly nonperturbative, showing the power of the QMC simulations.

\section{LOW-TEMPERATURE CRITICAL REGION}

Thus far, we have discussed the FM properties in the offcritical region. In this section, we further study the magnetic critical behavior through QMC. The FM order parameter is a conserved quantity, and thus, there are no quantum fluctuations; however, in 2D, thermal fluctuations are so strong that long-range FM ordering cannot appear at any finite temperatures for $\mathrm{SU}(2)$ symmetric models [58,59]. Nevertheless, magnetic properties still behave qualitatively differently in the off-critical and critical regions. We also consider the model in the Ising class in which true FM long-range ordering can appear and determine the renormalized Curie temperature $T_{c}$. 


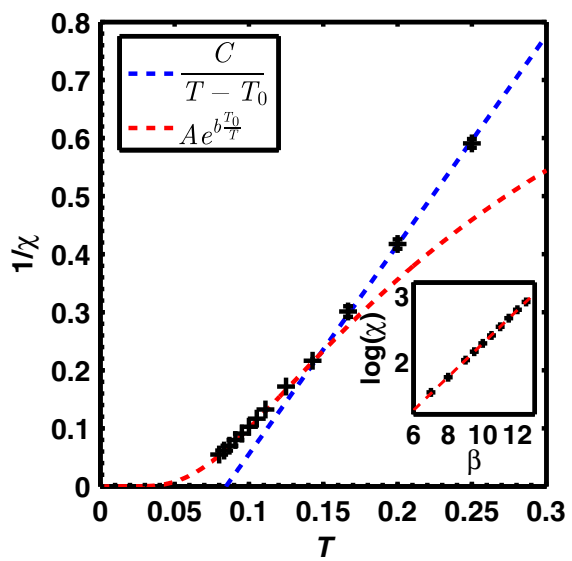

FIG. 6. The SU(2) invariant model in the critical region: $\chi^{-1}(T)$ at $n=1, J=2$, and $V=0$. It crosses over from the $\mathrm{CW}$ law to the exponential form of $\chi(T)=A e^{b\left(T_{0} / T\right)}$. The inset shows the linear scaling of $\ln \chi(T)$ versus $\beta$.

In spite of the quasi-1D band structure, the magnetic properties of our model are intrinsically 2D because Hund's interaction couples spins of different chains together and the total spin of each chain is not separately conserved. In Fig. 6, we present the crossover of $\chi^{-1}(T)$ from the offcritical region to the critical region based on the finite-size scaling presented in Appendix B. Although there is no distinct phase transition between the off-critical and critical regions, the temperature dependence of $\chi(T)$ changes qualitatively. The clear deviation from the $\mathrm{CW}$ law starts from $T \sim T_{0}=0.08$. In the critical region, the FM order parameter already develops a nonzero magnitude, and its directional fluctuations are described by the $\mathrm{O}(3)$ nonlinear $\sigma$ model. The FM correlation length increases exponentially as approaching zero temperature. $\chi(T)$ evolves to the exponential form fitted by $\chi=A e^{b\left(T_{0} / T\right)}[72,73]$, and the result in Fig. 6 shows $b=3.1 \pm 0.3$ at $n=1, V=0$, and $J=2$.

In order to obtain the FM long-range order, we modify Hund's coupling of Eq. (3) to reduce its symmetry from the $\mathrm{SU}(2)$ to the Ising class: We introduce $J_{\|}$and $J_{\perp}$ for the spin components in the $x y$ plane and along the $z$ direction, respectively, and choose $J_{\perp}>J_{\|}$. The $z$-component FM structure factor is defined as $S_{\perp}(T, L)=T \chi(T, L)$. For the case presented in Fig. $7(a)$, the finite size scaling of $S_{\perp}(T, L) / L^{2}$ yields the critical temperature $T_{c} \approx 0.134$. This result is also checked from the scaling in the critical region in Figs. 7(b) and 7(c). $S_{\perp} L^{-2+\eta}$ versus $T$ is plotted with $\eta=\frac{1}{4}$ from the anomalous dimension of the $2 \mathrm{D}$ Ising universal class. The crossings of curves yield the value of $T_{c}$ consistent with that of the previous scaling. Furthermore, a good data collapse is achieved by employing the scaling form

$$
S_{\perp} L^{-2+\eta}=f\left[\left(T-T_{c}\right) L^{1 / \nu}\right],
$$

with $\nu=1$ of the $2 \mathrm{D}$ Ising class.
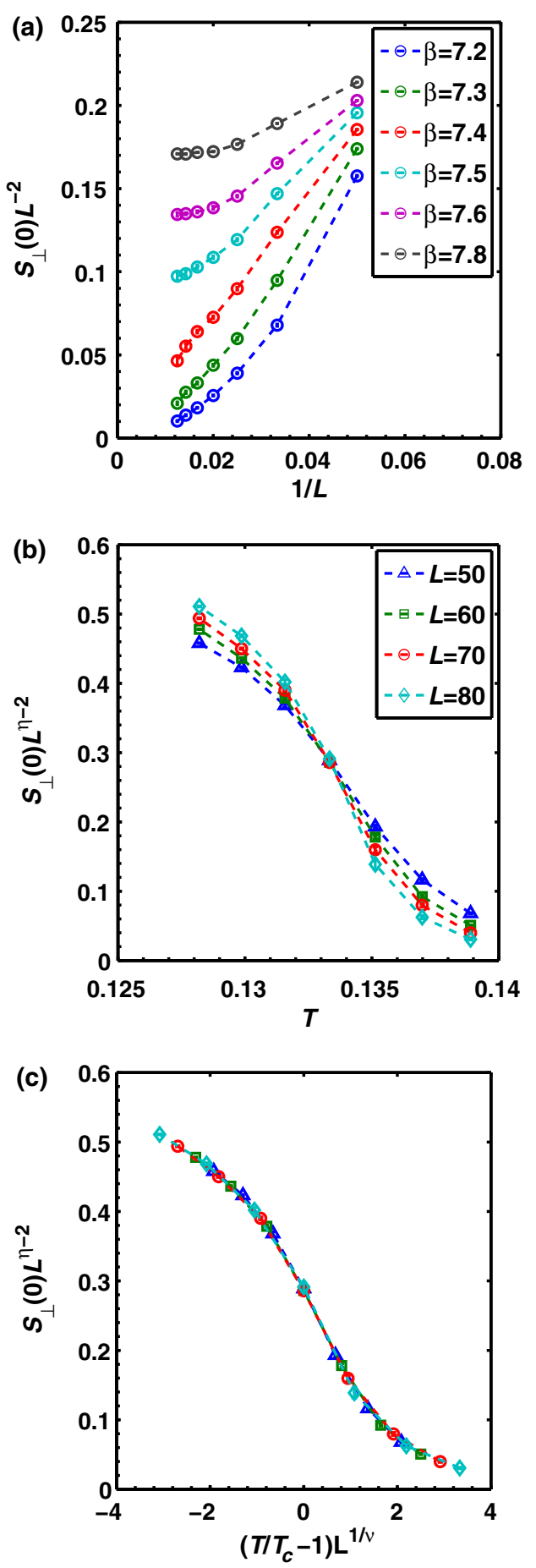

FIG. 7. The FM long-range ordering of the Ising symmetric model with parameters $J_{\perp}=2, J_{\|}=4, n=1$, and $V=0$. (a) The finite-size scaling of $S_{\perp} / L^{2}$. The FM critical temperature is extracted as $T_{c}=1 / \beta_{c}=0.134 \pm 0.002$, with $\beta_{c} \approx 7.4-7.5$. (b) The critical scaling: $S_{\perp} L^{-2+\eta}$ versus $T$, with $\eta=\frac{1}{4}$. The crossing of curves at different values of $L$ yields $T_{c} \approx 0.134$. (c) The data collapse of the scaling form $S_{\perp}(0) L^{-2+\eta}=$ $f\left[\left(T-T_{c}\right) L^{1 / \nu}\right]$ fitted by the parameters of $\nu=1$, $\eta=\frac{1}{4}$, and $T_{c}=0.1337$. The error bars are typically smaller than symbols. 
In Appendix $\mathrm{D}$, the mean-field value $T_{0} \approx 0.20$ is extracted based on the extrapolation of the CW behavior. Compared to the mean-field value $T_{0}, T_{c}$ is about $67 \%$ of $T_{0}$ as a result of the critical non-Gaussian fluctuations. For the 2D Ising mode with only nearest-neighbor coupling on the square lattice, the Onsager solution gives rise to $T_{c}=2 / \ln (\sqrt{2}+1) \approx 2.269$, which is $57 \%$ of the BraggWilliams mean-field results $T_{0}=4$. Thus, the critical fluctuation strength of the case presented in Fig. 7 is weaker compared to that in the 2D Ising model in spite of the effect of the transverse component $J_{\|}$. This is due to the itinerant nature of our model such that the effective FM coupling is beyond two nearest-neighboring sites.

\section{DISCUSSIONS ON THE PHYSICS OF FINITE $t_{\perp}$ AND $U$}

In this section, we discuss the situations when the conditions for the absence of the sign problem are loosed, including the presence of a small transverse hopping $t_{\perp}$ term, as shown in Eq. (6), and the case of finite values of $U$.

\section{A. QMC simulations with small transverse hopping term}

The presence of the $t_{\perp}$ term enables electrons moving in the entire two-dimensional lattice; thus, the fermion sign problem does appear. Nevertheless, the sign problem is not severe at small values of $t_{\perp}$, such that QMC simulations can still be performed. In Fig. 8, the average of the sign is calculated from $t_{\perp}=0$ to 0.05 at small and intermediate sample sizes with $\beta=6$. We use the periodical boundary condition for the entire system, which is different from the boundary condition used in previous calculations, in order to eliminate the sign problem when electrons hop across the boundary. The previous boundary condition is feasible at $t_{\perp}=0$ because the particle number in each chain is conserved, as explained in Sec. II B. Now, under the periodical boundary condition, even at $t_{\perp}=0$ the sign is

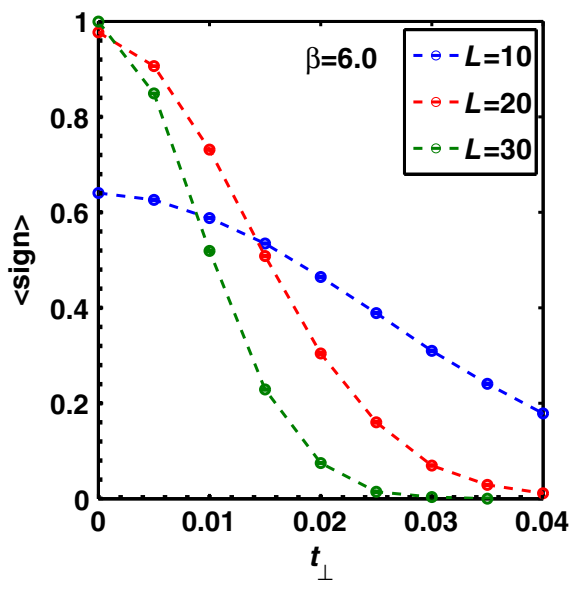

FIG. 8. The average of the fermion sign versus $t_{\perp}$ at different values of $L$. The parameters are $V=0, J=2, n=1$, and $\beta=6$. not positive definite: when one electron hops across the boundary, if the fermion number in that chain is an even number, the matrix element acquires an extra sign. This boundary effect is more prominent at small sample sizes (e.g., $L=10$ ) but already becomes negligible at intermediate sample sizes, say, $L>20$. As $t_{\perp}$ deviates from 0 , the 2D motion of electrons suppresses the average sign and it drops more rapidly at larger sample sizes.

We simulate the spin susceptibility and present its inverse $\chi^{-1}(T)$ in Fig. 9, with $t_{\perp}=0.02$. The results at $t_{\perp}=0$ under the periodical boundary condition are also plotted for comparison. An intermediate sample size ( $L=20)$ is used and the simulation is performed from the high to intermediate temperature regions. The results at $t_{\perp}=0.02$ are nearly the same as those at $t_{\perp}=0$, which still exhibit the $\mathrm{CW}$ behavior. At the lowest temperature simulated $\beta=6$, the average sign at $t_{\perp}=0.02$ is already significantly below 1 . Nevertheless, the difference between $\chi^{-1}(T)$ at $t_{\perp}=0$ and 0.02 remains negligible. These results show that the magnetic properties are not so sensitive to $t_{\perp}$ when $t_{\perp} / t_{\|} \ll 1$.

Certainly, when $t_{\perp}$ reaches the same order as $t_{\|}$, the band structure will become genuinely two dimensional. In this case, the previous ground FM theorem does not apply, and a quantum phase transition is likely to occur from the FM to paramagnetic ground states. Unfortunately, the sign problem will be very severe and, thus, reliable QMC simulations cannot be performed. It would be interesting to further develop other analytic and numeric methods to investigate this problem.

\section{B. Effect of the finite $\boldsymbol{U}$}

As explained in Sec. II B, the many-body bases for simulations, which are also used for the proof of FM ground-state theorems in Ref. [21], are constructed by ordering electrons according to their locations along one

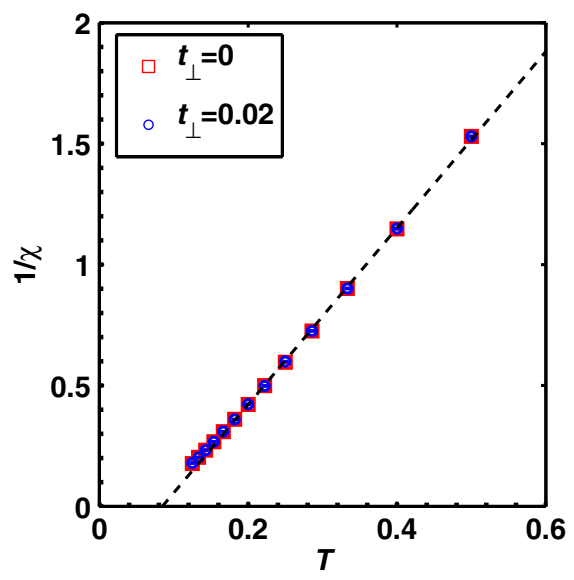

FIG. 9. $\chi^{-1}(T)$ at $t_{\perp}=0$ and 0.02 , respectively, with the system size $L=20$. Periodical boundary conditions are used. The parameters are $V=0, J=2, n=1$. 
chain by another regardless of their spin configurations. This set of bases is convenient to accommodate to the spinflip term of Hund's coupling to be free of the sign problem; nevertheless, finite $U$ does cause this problem. If $U$ is finite, states with doubly occupied orbitals are allowed, and electrons with opposite spins can exchange their locations, which causes the sign problem. This situation is different from the 1D single-band Hubbard model, in which finite $U$ does not lead to the sign problem. This is because the usual single-band Hubbard interaction does not contain spin-flip terms, and the many-body bases can be constructed as direct products of states in spin-up and -down sectors separately. We defer the QMC simulations for the multiorbital model with finite $U$ to a later publication, but briefly analyze the physical effect below.

Basically, a large but finite $U$ introduces an antiferromagnetic (AFM) energy scale of $J_{\mathrm{AFM}}=4 t_{\|}^{2} / U$ for two electrons lying in adjacent sites in the same chain. Its effect in the low-electron-density region is unimportant but becomes important in the limit of $n \rightarrow 2$ in which most sites are occupied as spin-1 moments. In this region, the FM energy scale $T_{0}(n)$ is suppressed because of the low density of mobile holes, and finally, it becomes weaker than $J_{\mathrm{AFM}}$. Consequently, we expect a ground-state phase transition at a critical density $n_{c}$ close to $n=2$, which marks a transition from the FM ordering at $n<n_{c}$ to the AFM ordering at $n_{c}<n<2$. Nevertheless, if $U$ is sufficiently weak, the system becomes weakly correlated and the FM ordering could be completely suppressed even in the presence of Hund's coupling.

\section{EXPERIMENT REALIZATIONS}

The QMC simulations presented above are not only of academic interest but also provide new directions to explore new FM materials in various physical systems, including both the ultracold atom optical lattices and the strongly correlated transition-metal oxides.

Recently, the study of itinerant FM states has become a research focus in ultracold cold atom physics [24-31]. However, so far, it is still in debate whether the experiment results based on the upper branches of the Feshbach resonances have shown the existence of itinerant FM or not. Our work suggests a new direction for the further experimental exploration of itinerant FM in the high orbital bands in optical lattices. Our band Hamiltonian can be accurately implemented in the $p$-orbital band in the ultracold atom optical lattices [74-76]. Because of the anisotropy of $p$-orbital orientation, the transverse $\pi$ bonding amplitude $t_{\perp}$ is usually much smaller than the longitudinal $\sigma$-bonding $t_{\|}$. The ratio of $t_{\perp} / t_{\|}$decreases as increasing the optical potential depth $V_{0}$. As shown in Ref. [74], as $V_{0} / E_{R}=15$, where $E_{R}$ is the recoil energy of the laser forming the optical lattice, $t_{\perp} / t_{\|} \approx 5 \%$, such that we can neglect the $t_{\perp}$ term in Eq. (2). Furthermore, the interaction strength is also tunable in optical lattices by simply varying laser intensities, and the strong coupling regime can be reached. A variation study based on the Gutzwiller projection also shows that the ground-state FM may start from intermediate coupling strength [76]. Our simulations on the thermodynamic properties provide important guidance for future experiments.

Our work is also helpful for the current effort of searching for novel FM materials in transition-metal oxides, in particular, in systems with the $t_{2 g}$-orbital bands, i.e., $d_{x z}, d_{y z}$, and $d_{x y}$ bands, with the quasi-2D layered structure. In fact, FM has been observed experimentally in the (001) interface of $3 d$-orbital transition-metal oxides such as $\mathrm{SrTiO}_{3} / \mathrm{LaAlO}_{3}$ [20,77-80], which has been a recent research focus in condensed-matter physics. The dispersions of $d_{x z}$ and $d_{y z}$-orbital bands are also highly anisotropic, i.e., the longitudinal bonding parameter $t_{\|}$is much larger than the transverse one $t_{\perp}$, as described in Eq. (2), by replacing $p_{x(y)}$ with $d_{x(y) z}$. The on-site repulsive interaction of the $3 d$ electrons is particularly strong, such that the projection of doubly occupied orbitals is a good approximation.

Even though there is an additional quasi-2D $d_{x y}$-orbital band in the $\mathrm{SrTiO}_{3} / \mathrm{LaAlO}_{3}$ interfaces, which is presumably paramagnetic by itself, it is conceivable that the overall system remains FM as shown in experiments, and our results still apply qualitatively. The reason is that the quasi1D bands $d_{x(y) z}$ do not hybridize with the quasi-2D $d_{x y}$ band by the nearest-neighbor hopping due to their different parity eigenvalues under the reflections with respect to $x y$, $y z$, and $z x$ planes, respectively. It is a good approximation that the particle numbers in the $d_{x y}$ band and in the $d_{x(y) z}$ bands are separately conserved, and they only couple through interactions. The coupling is ferromagnetic by nature due to Hund's rule. Since the quasi-1D bands by themselves are already FM in the strong-coupling regime, their coupling to the paramagnetic $d_{x y}$ band acts as using a permanent ferromagnet to polarize a paramagnet, and it is conceivable that overall the ferromagnetism is enhanced.

\section{CONCLUSIONS}

In summary, we nonperturbatively investigate the thermodynamic properties of an unambiguous itinerant FM system with multiorbital structures through the method of the SSE QMC. The simulations are proved to be signproblem-free in all the electron density region, and thus, reliable numerical results can be obtained at high numeric accuracy. Because of the nature of asymptotic exactness of our simulations, they provide a solid reference point for the study of the strong correlation effects of the thermodynamic properties of itinerant FM systems. There is a wide temperature region $T_{0}<T<T_{\mathrm{ch}}$, in which the spin channel is incoherent without local moments existing as a priori, while the charge channel exhibits metallic behavior. The spin magnetic susceptibility exhibits the $\mathrm{CW}$ law in the off-critical region as a result of strong correlations. It further crosses over to the exponential growth in the critical region. The compressibility is weakly temperature 
dependent and saturates to its zero-temperature value. The true FM long-range transition appears when the symmetry class is reduced from $\mathrm{SU}(2)$ to Ising. The finite-size scaling in the critical region gives rise to an accurate determination of the FM transition temperature. Our work is also closely related to the experimental efforts of searching for novel FM states of matter in both ultracold atom optical lattices and $3 d$ transition-metal oxide materials.

\section{ACKNOWLEDGMENTS}

C.W. is grateful for the hospitality of Center of Mathematical Sciences and Applications at Harvard University where part of the work was done. S.X. and C. W. are supported by the NSF DMR-1410375 and AFOSR FA9550-14-1-0168. Y. L. is grateful for support from the Princeton Center for Theoretical Science. S.X., Y. L., and C. W. thank D. Arovas, X. Dai, J. E. Hirsch, M. Randeria, A. W. Sandvik, H. Shao, L. J. Sham, D. J. Singh, N. Trivedi, and $\mathrm{Lu} \mathrm{Yu}$ for helpful discussions. All the simulations were performed on Tiger Cluster in Princeton. C.W. also acknowledges support from the National Natural Science Foundation of China under Grant No. 11328403, the CAS/ SAFEA International Partnership Program for Creative Research Teams of China, and President's Research Catalyst Award No. CA-15-327861 from the University of California Office of the President. Y.L. thanks the hospitality of the Aspen Center of physics under the support of NSF Grant No. PHYS-1066293.

\section{APPENDIX A: PARAMETERS FOR QMC SIMULATIONS}

We use the SSE method of QMC to simulate the Hamiltonian Eq. (2) plus Eq. (3) in the main text with the directed loop algorithm [64-68]. A Monte Carlo step is defined as a diagonal vertex update followed by many directed off-diagonal loop updates to ensure that most of vertex legs are visited by the worm head. The simulated system size of the square lattice is $L \times L$ with the values of $L$ given in each figure that depends on $L$. The simulations are run in parallel on 16 cores. On each core, $1.5 \times 10^{5}$ warm-up steps are used, and for a typical data point, we use $10^{5}$ QMC steps and perform $10^{4}$ measurements. For the simulation with the largest system size $(L=80), 3.0 \times 10^{5}$ warm-up steps and $2.0 \times 10^{5}$ QMC steps are used.

In order to maintain ergodicity, the directed loop algorithm is carried out in the grand canonical ensemble in which the chemical potential $\mu$ is the characteristic variable. Nevertheless, in realistic systems, it is more natural to fix the average fermion number per site $n$ rather than to fix $\mu$. For example, in a system with a fixed average value of $n, \mu(T)$ changes as varying the temperature $T$. Therefore, in presenting the simulation results for a fixed value of $n$, we carefully adjust $\mu$ to maintain $n$ invariant. The obtained relations of $\mu(T)$ at various values of $n$ with the system size $L=8$ are plotted in Fig. 10. The
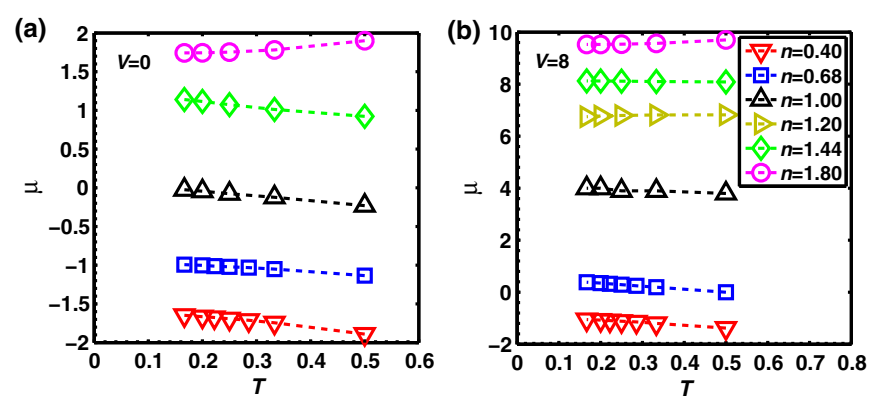

FIG. 10. The chemical potential $\mu(T)$ is tuned to maintain the filling $n$ unchanged at all temperatures. $V=0$ in (a), and $V=8$ in (b). The system size is $L=8$.

dependence of $\mu$ on $L$ is weak, and thus, we use the same set values of $\mu(T)$ for even larger sample sizes, except the case of $V=0$ and $n=0.40$. In this case, the finite-size effect of $\mu$ is relatively strong, and we calculate $\mu(T)$ at $L=10$ and use it for larger sample sizes. By this method, we can maintain the values of $n$ invariant within the error of $\Delta n=0.006$ for all the simulations.

\section{APPENDIX B: MORE INFORMATION ON THERMODYNAMIC PROPERTIES}

In Figs. 11 and 12, the finite-size scaling of $\chi(T, L)$ are plotted with $J=2$ at $V=0$ and $V=8$, respectively. In
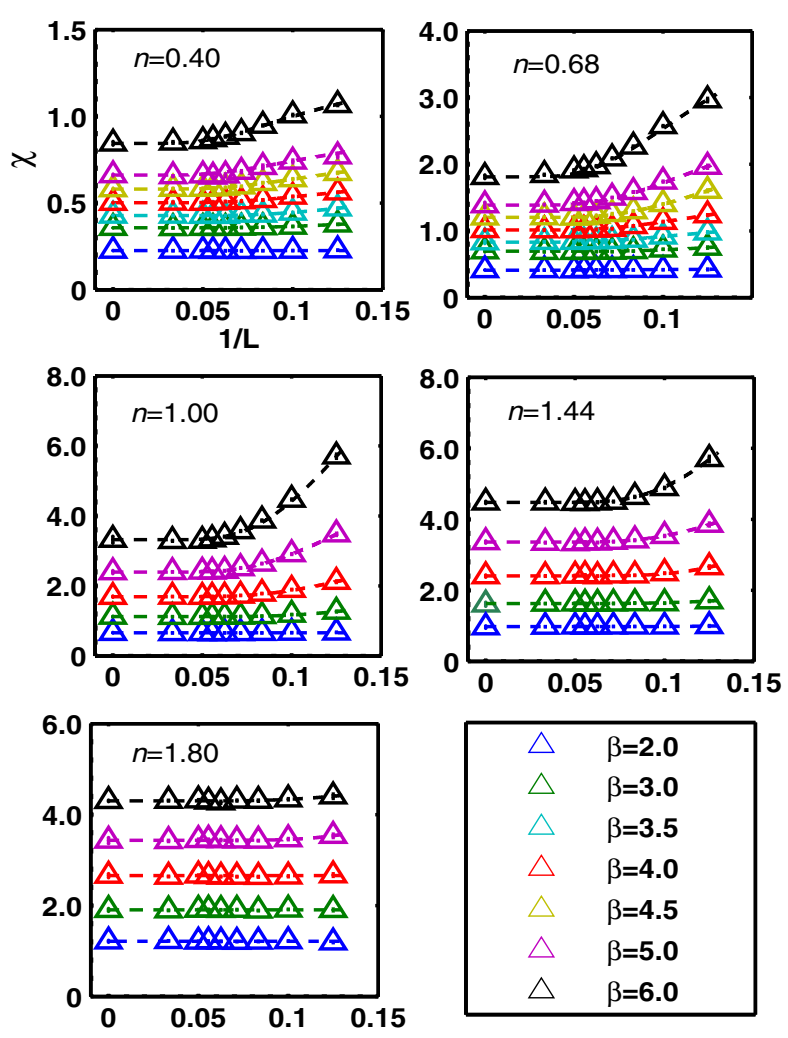

FIG. 11. The finite-size scalings for $\chi(T, L)$ versus $1 / L$ with the fitting $\chi(T, L)=a L e^{-L / b}+\chi(T)$. Values of $\beta=1 / T$ are presented in the legend. The parameter value at $V=0$ and $J=2$. 

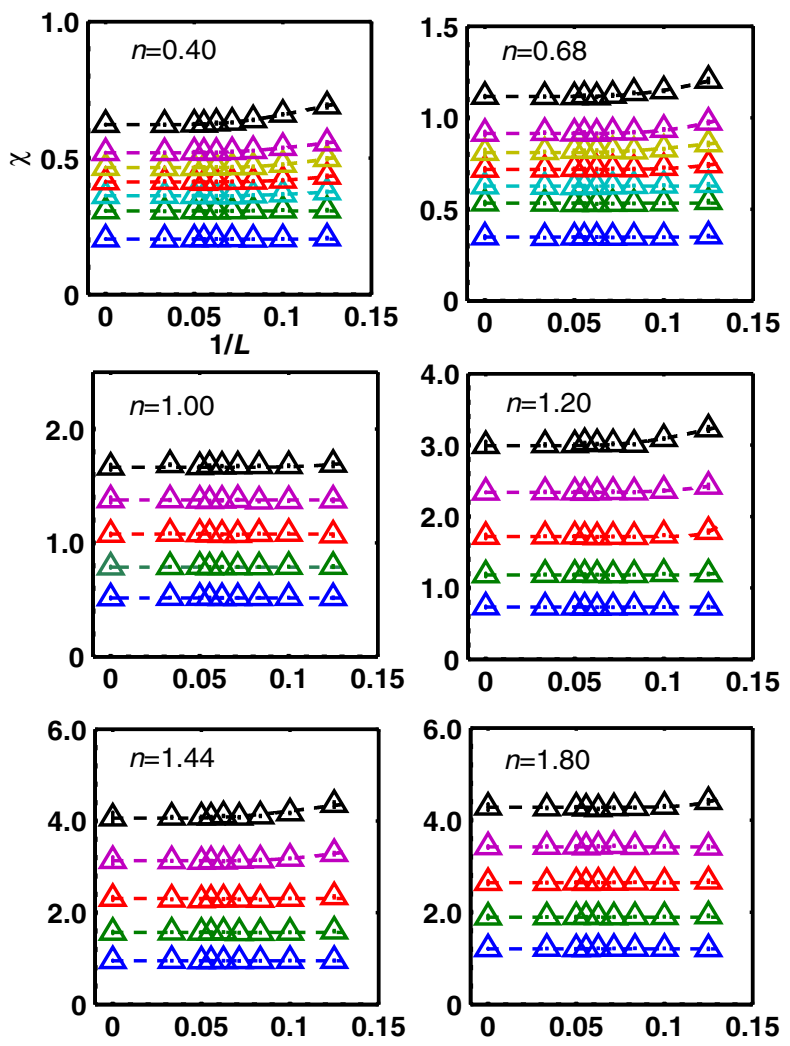

FIG. 12. The finite -size scaling of $\chi(T, L)$ versus $1 / L$ for $V=8$. Scaling ansatz $\chi(T, L)=a L e^{-L / b}+\chi(T)$ is used. The legend is the same as in Fig. 11.

both cases, curves are fitted with the scaling ansatz $\chi(L, T)=\chi_{0}(T)+a L e^{-L / b}$ to extrapolate the spin susceptibility $\chi(T)$ for the infinite system size. For the data sets with $\beta<6$, we simulate lattice sizes $L \times L$ up to $L=30$.

In Fig. 13, we present the finite-size scaling of $\chi(T, L)$ in the critical region with $\beta$ from 7 to 12.5 and with

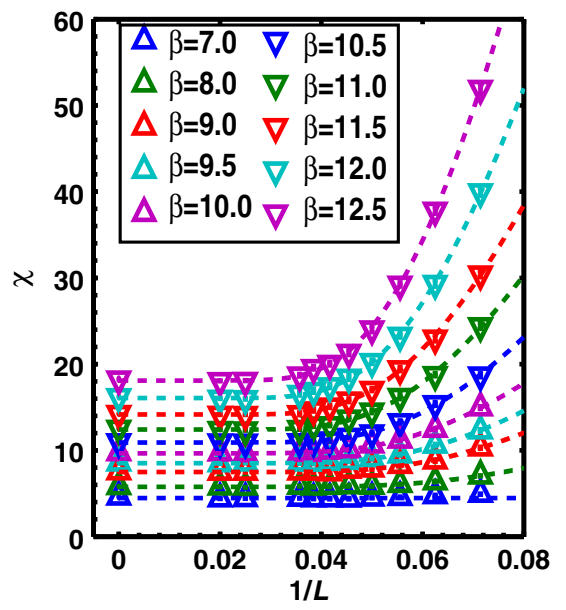

FIG. 13. The finite-size scaling of $\chi(T, L)$ at $n=1, V=0$, and $J=2$ in the low-temperature critical region with the scaling ansatz $\chi(T, L)=a L e^{-L / b}+\chi(T)$ used. Values of $\beta=1 / T$ are presented in the legend.

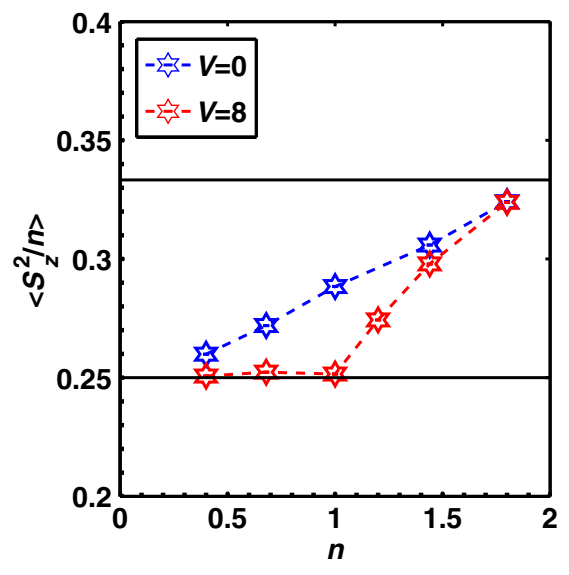

FIG. 14. The QMC results for the normalized on-site spin moment $\left\langle S_{z}^{2}\right\rangle / n$ at $\beta=6, J=2$, and $L=30$. Values of $V$ are marked in the inset.

parameters $V=0, J=2$, and $n=1$. The extrapolated values of $\chi(T)$ are used in Fig. 3(a). Let us look at the curve with $\beta=10$, the dependence of $\chi(T, L)$ on $L$ converges at large values of $L$. The starting of convergence takes places at values of $L$ at the order from 10 to 20, and thus, we estimate the FM correlation length $\xi$ at $\beta=10$ also in this range.

In Fig. 14, we plot the QMC simulation results for the on-site spin moment square normalized by filling density $\left\langle S_{z}^{2}\right\rangle / n$, which behaves nearly the same as the Curie constants presented in Fig. 3(b). Because of the $\mathrm{SU}(2)$ symmetry, the on-site spin moment square $\left\langle S^{2}\right\rangle / n=3\left\langle S_{z}^{2}\right\rangle / n$. A discussion on $\left\langle S_{z}^{2}\right\rangle / n$ is presented in the Sec. III C.

Next, we discuss the momentum space distribution $n_{F}(k)$ which is $1 \mathrm{D}$-like because the particle number of each chain is separately conserved. Because of this feature, we make a special choice of boundary conditions to remove the sign problem: the periodical (antiperiodical) boundary condition for a chain if its particle number is odd (even). Our simulation uses the grand canonical assemble, and
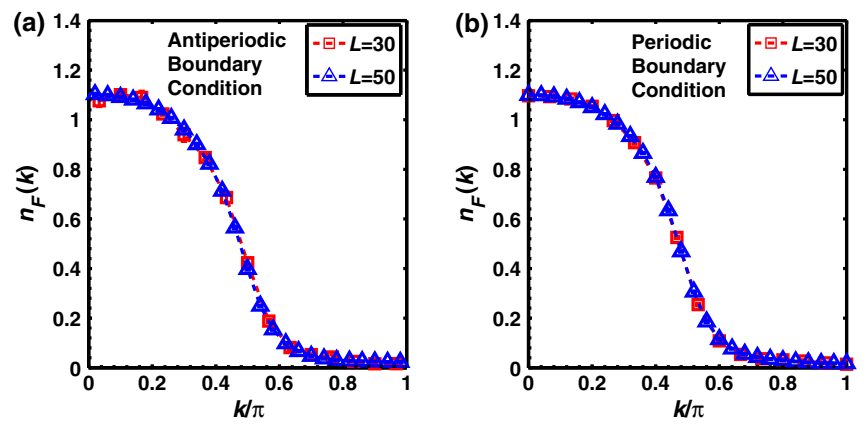

FIG. 15. The momentum space occupation $n_{F}(k)$ at $\beta=10$, $V=0, J=2$, and $n=1$. Results of $n_{F}(k)$ for both the (a) antiperiodical boundary condition and (b) periodical boundary condition are presented at two different sizes of $L=30$ and 50 . 
thus, both configurations with even and odd particle numbers are sampled, which can also be easily distinguished. For configurations with odd particle numbers, the values of $k$ take $2 p \pi / L$ with $p=0, \pm 1, \ldots \pm(L-1)$, and for configurations with even particle numbers, the values of $k$ take $2 p \pi / L$ with $p= \pm \frac{1}{2}, \ldots, \pm\left(L-\frac{1}{2}\right)$. For this reason, we simulate $n_{F}(k)$ by separately sampling configurations of even and odd particle numbers and present both of them in Figs. 15(a) and 15(b), respectively. Results of the sample sizes with $L=30$ and 50 are presented, which shows that the finite-size dependence is very weak. At both sample sizes $L=30$ and 50 , the differences caused by using periodical or antiperiodical boundary conditions are rather small, and the result under the periodical boundary condition is presented Fig. 1(c) in the main text.

\section{APPENDIX C: ORBITAL ORDERING AT THE COMMENSURATE FILLING $N=1$}

Here, we present the QMC simulations on the orbital ordering with a large value of the interorbital repulsion $V$. Large $V$ suppresses doubly occupied on-site states, and at the commensurate filling $n=1$, the ground state is in the Mott-insulating state. In this case, fermions become local moments. At zero temperature, even though electron spins are fully polarized, the orbital degree of freedom enables the superexchange in the orbital channel [81]. The orbital exchange is described by an antiferro-orbital Ising model

$$
H_{\mathrm{ex}}=J_{\mathrm{orb}} \sum_{\vec{r}, \vec{r}^{\prime}} \tau_{z}(\vec{r}) \tau_{z}\left(\vec{r}^{\prime}\right)
$$

where $J_{\text {orb }}=t_{\|}^{2} / V$ and $\tau_{z}=p_{x}^{\dagger} p_{x}-p_{y}^{\dagger} p_{y}$. At low temperatures, due to the prominent FM tendency, the above orbital

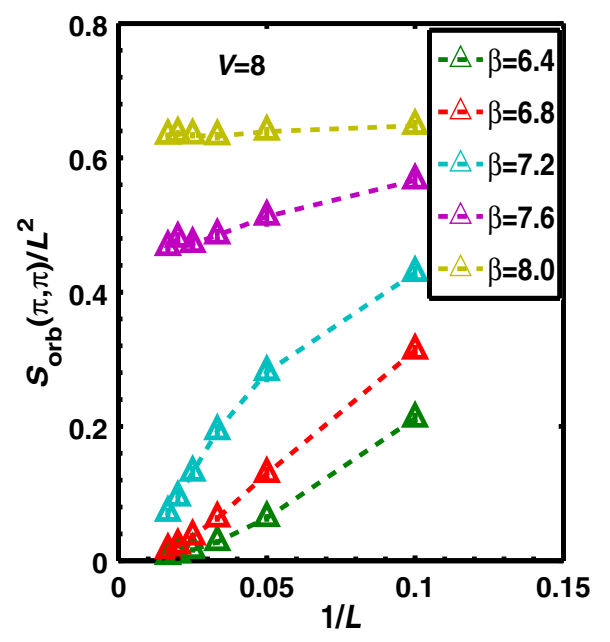

FIG. 16. The finite-size scaling of $S_{\text {orb }}(\pi, \pi) / L^{2}$ with the parameter values $J_{\perp}=J_{\|}=2, n=1$, and $V=8$. The system sizes range from $L=10$ to 60 , and the values of inverse temperature $\beta$ are presented in the legend. The orbital ordering occurs at $\beta$ between 7.2 and 7.6, which corresponds to $T_{\text {orb }} / t_{\|} \approx 0.132-0.139$. exchange model still applies. Thus, below the temperature scale around $J_{\text {orb }}$, the antiferro-orbital ordering, i.e., the staggered occupation of $p_{x}$ and $p_{y}$ orbitals, will appear.

We define the equal-time orbital structure factor as

$S_{\mathrm{orb}}(\vec{q}, \tau)=\frac{1}{L^{2}} \sum_{\vec{r}_{1}, \vec{r}_{2}}\left\langle m_{\mathrm{orb}}\left(\vec{r}_{1}, \tau\right) m_{\mathrm{orb}}\left(\vec{r}_{2}, \tau\right)\right\rangle e^{i \vec{q} \cdot\left(\vec{r}_{2}-\vec{r}_{1}\right)}$,

where $m_{\text {orb }}(\vec{r}, \tau)=n_{x}(\vec{r}, \tau)-n_{y}(\vec{r}, \tau)$ is the on-site orbital polarization. Since the orbital ordering occurs at the wave vector $(\pi, \pi)$, we present the QMC simulation of the finite scaling of $S_{\text {orb }}(\pi, \pi) / L^{2}$ in Fig. 16. It indicates that the antiferro-orbital ordering appears at low temperatures, and the critical temperature $T_{\text {orb }} / t_{\|}$lies between 0.132 and 0.139 .

\section{APPENDIX D: QMC SIMULATIONS FOR THE ISING-CLASS HAMILTONIAN}

For the Ising-class Hamiltonian with the modified Hund's coupling with $J_{\perp}=2 J_{\|}=4$, we extract its
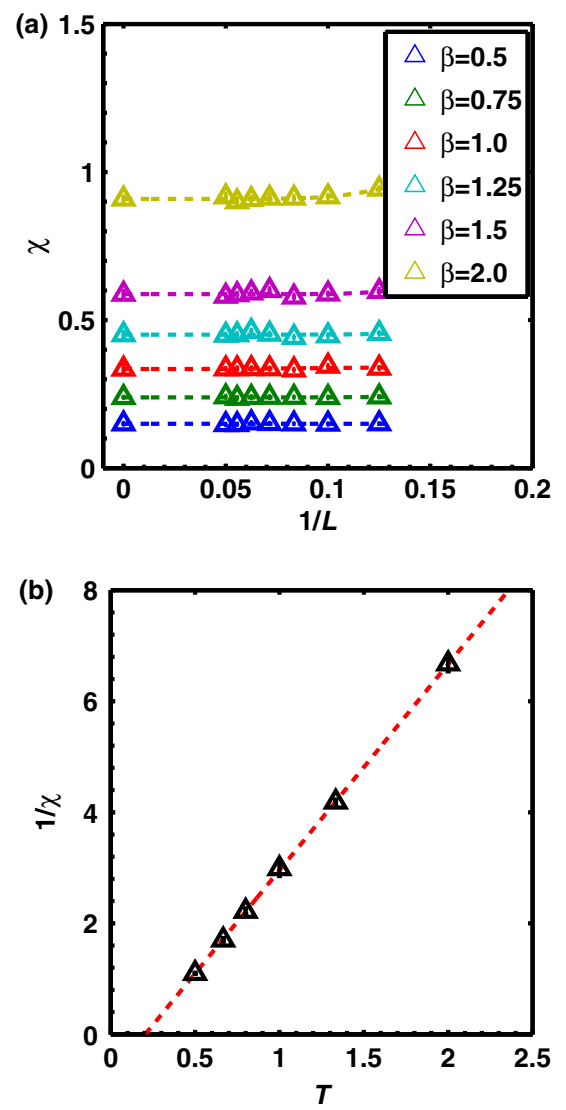

FIG. 17. (a) The finite-size scaling of $\chi(T, L)$ versus $1 / L$ in the off-critical region. Hund's rule coupling is modified as $J_{\perp}=2 J_{\|}=4$. (b) The $\mathrm{CW}$ behavior of the extrapolated $\chi^{-1}(T)$. The interception on the temperature axis yields $T_{0} / t_{\|}=0.20 \pm 0.01$. 
mean-field value of the Curie temperature $T_{0}$ following the method presented in the main text. The spin susceptibility $\chi(T)$ is obtained after the finite-size scaling for $\chi(T, L)$ in the off-critical region as shown in Fig. 17(a). By the linear extrapolation of $\chi^{-1}(T)$ in the off-critical region, we obtain $T_{0}$ from the interception of $\chi^{-1}(T)$ on the temperature axis as shown in Fig. 17(b). The scaling is performed in the region $T>0.5$ below which the deviation from the $\mathrm{CW}$ behavior appears. The linear extrapolation of $\chi^{-1}(T)$ gives rise to $T_{0}=0.20 \pm 0.01$. The magnetic structure factors at even lower temperatures in the critical region are presented in Fig. 7.

[1] E. C. Stoner, Collective electron ferromagnetism, Proc. R. Soc. A 165, 372 (1938).

[2] C. Zener, Interaction between the $d$-shells in the transition metals. II. Ferromagnetic compounds of manganese with perovskite structure, Phys. Rev. 82, 403 (1951).

[3] J.C. Slater, Ferromagnetism and the band theory, Rev. Mod. Phys. 25, 199 (1953).

[4] P. W. Anderson and H. Hasegawa, Considerations on double exchange, Phys. Rev. 100, 675 (1955).

[5] E. H. Lieb and D. Mattis, Theory of ferromagnetism and the ordering of electronic energy levels, Phys. Rev. 125, 164 (1962).

[6] Y. Nagaoka, Ferromagnetism in a narrow, almost half-filled s band, Phys. Rev. 147, 392 (1966).

[7] C. Herring, Magnetism (Academic, Berlin, 1966).

[8] J. A. Hertz, Quantum critical phenomena, Phys. Rev. B 14, 1165 (1976).

[9] K. K. Murata and S. Doniach, Theory of Magnetic Fluctuations in Itinerant Ferromagnets, Phys. Rev. Lett. 29, 285 (1972).

[10] T. Moriya, Spin Fluctuations in Itinerant Electron Magnetism, Springer Series in Solid-State Sciences Vol. 56 (Springer, Berlin, 1985).

[11] J. E. Hirsch, Metallic ferromagnetism in a single-band model, Phys. Rev. B 40, 2354 (1989).

[12] A. Mielke and H. Tasaki, Ferromagnetism in the Hubbard model-examples from models with degenerate singleelectron ground states, Commun. Math. Phys. 158, 341 (1993).

[13] H. Tasaki, Ferromagnetism in the Hubbard Models with Degenerate Single-Electron Ground States, Phys. Rev. Lett. 69, 1608 (1992).

[14] A. J. Millis, Effect of a nonzero temperature on quantum critical points in itinerant fermion systems, Phys. Rev. B 48, 7183 (1993).

[15] Band-Ferromagnetism, edited by K. Baberschke, W. Noting, and M Donath (Springer-Verlag, Berlin, 2001).

[16] D. Belitz, T. R. Kirkpatrick, and T. Vojta, How generic scale invariance influences quantum and classical phase transitions, Rev. Mod. Phys. 77, 579 (2005).

[17] J. Jackiewicz and K. S. Bedell, Quantum fluctuation driven first-order phase transition in weak ferromagnetic metals, Philos. Mag. 85, 1755 (2005).
[18] H. v. Löhneysen, A. Rosch, M. Vojta, and P. Wölfle, Fermiliquid instabilities at magnetic quantum phase transitions, Rev. Mod. Phys. 79, 1015 (2007).

[19] D. L. Maslov and A. V. Chubukov, Nonanalytic paramagnetic response of itinerant fermions away and near a ferromagnetic quantum phase transition, Phys. Rev. B 79, 075112 (2009).

[20] G. Chen and L. Balents, Ferromagnetism in Itinerant TwoDimensional $t_{2 g}$ Systems, Phys. Rev. Lett. 110, 206401 (2013).

[21] Y. Li, E. H. Lieb, and C. Wu, Exact Results for Itinerant Ferromagnetism in Multiorbital Systems on Square and Cubic Lattices, Phys. Rev. Lett. 112, 217201 (2014).

[22] Y. Sang, D. Belitz, and T. R. Kirkpatrick, Disorder Dependence of the Ferromagnetic Quantum Phase Transition, Phys. Rev. Lett. 113, 207201 (2014).

[23] D. C. Mattis, The Theory of Magnetism Made Simple (World Scientific, Singapore, 2006).

[24] R. A. Duine and A. H. MacDonald, Itinerant Ferromagnetism in an Ultracold Atom Fermi Gas, Phys. Rev. Lett. 95, 230403 (2005).

[25] G.-B. Jo, Y.-R. Lee, J.-H. Choi, C. A. Christensen, T. H. Kim, J. H. Thywissen, D. E. Pritchard, and W. Ketterle, Itinerant ferromagnetism in a fermi gas of ultracold atoms, Science 325, 1521 (2009).

[26] S. Zhang, H.-h. Hung, and C. Wu, Proposed realization of itinerant ferromagnetism in optical lattices, Phys. Rev. A 82, 053618 (2010).

[27] I. Berdnikov, P. Coleman, and S. H. Simon, Itinerant ferromagnetism in an atom trap, Phys. Rev. B 79, 224403 (2009).

[28] D. Pekker and E. Demler, Competing Instabilities in Quench Experiments with Ultracold Fermi Gases near a Feshbach Resonance, arXiv:1107.3930.

[29] C.-C. Chang, S. Zhang, and D. M. Ceperley, Itinerant ferromagnetism in a fermi gas with contact interaction: Magnetic properties in a dilute Hubbard model, Phys. Rev. A 82, 061603 (2010).

[30] X. Cui and T.-L. Ho, Ground-state ferromagnetic transition in strongly repulsive one-dimensional fermi gases, Phys. Rev. A 89, 023611 (2014).

[31] S. Pilati, I. Zintchenko, and M. Troyer, Ferromagnetism of a Repulsive Atomic Fermi Gas in an Optical Lattice: A Quantum Monte Carlo Study, Phys. Rev. Lett. 112, 015301 (2014).

[32] W. Kohn and L. J. Sham, Self-consistent equations including exchange and correlation effects, Phys. Rev. 140, A1133 (1965).

[33] U. V. Barth and L. Hedin, A local exchange-correlation potential for the spin polarized case. I, J. Phys. C 5, 1629 (1972).

[34] V. L. Moruzzi, J. F. Janak, and A. R. Williams, Calculated Electronic Properties of Metals (Pergamon, New York, 1978).

[35] B. Himmetoglu, A. Floris, S. de Gironcoli, and M. Cococcioni, Hubbard-corrected DFT energy functionals: The LDA $+U$ description of correlated systems, Int. J. Quantum Chem. 114, 14 (2014).

[36] A. Georges, G. Kotliar, W. Krauth, and M. J. Rozenberg, Dynamical mean-field theory of strongly correlated fermion 
systems and the limit of infinite dimensions, Rev. Mod. Phys. 68, 13 (1996).

[37] E. Gull, A. J. Millis, A. I. Lichtenstein, A. N. Rubtsov, M. Troyer, and P. Werner, Continuous-time Monte Carlo methods for quantum impurity models, Rev. Mod. Phys. 83, 349 (2011).

[38] K. Held, I. A. Nekrasov, G. Keller, V. Eyert, N. Blümer, A. K. McMahan, R. T. Scalettar, T. Pruschke, V. I. Anisimov, and D. Vollhardt, The LDA + DMFT Approach to Materials with Strong Electronic Correlations, arXiv: cond-mat/0112079.

[39] M. C. Gutzwiller, Effect of Correlation on the Ferromagnetism of Transition Metals, Phys. Rev. Lett. 10, 159 (1963).

[40] J. N. Zhuang, L. Wang, Z. Fang, and X. Dai, Fast impurity solver based on Gutzwiller variational approach, Phys. Rev. B 79, 165114 (2009).

[41] W. Weber, J. Bünemann, and F. Gebhard, BandFerromagnetism (Springer-Verlag, Berlin, 2001).

[42] B. S. Shastry, H. R. Krishnamurthy, and P. W. Anderson, Instability of the Nagaoka ferromagnetic state of the $u=\infty$ Hubbard model, Phys. Rev. B 41, 2375 (1990).

[43] G.-S. Tian, Stability of the Nagaoka State in the one-band Hubbard model, Phys. Rev. B 44, 4444 (1991).

[44] H. Tasaki, Extension of Nagaoka's theorem on the large- $U$ Hubbard model, Phys. Rev. B 40, 9192 (1989).

[45] L. Liu, H. Yao, E. Berg, S. R. White, and S. A. Kivelson, Phases of the Infinite U Hubbard Model on Square Lattices, Phys. Rev. Lett. 108, 126406 (2012).

[46] Z.-C. Gu, H.-C. Jiang, and G. Baskaran, Emergence of $p+i p$ Superconductivity in 2D Strongly Correlated Dirac Fermions, arXiv:1408.6820.

[47] A. Mielke, Ferromagnetic ground states for the Hubbard model on line graphs, J. Phys. A 24, L73 (1991).

[48] Y. Li, Exact results for itinerant ferromagnetism in a $t_{2 g}$ orbital system on cubic and square lattices, Phys. Rev. B 91, 115122 (2015).

[49] T. Moriya and A. Kawabata, Effect of spin fluctuations on itinerant electron ferromagnetism, J. Phys. Soc. Jpn. 34, 639 (1973).

[50] J. Stoehr and H. C. Siegmann, Magnetism, From Fundamentals to Nanoscale Dynamics (Springer, New York, 2006).

[51] P Fazekas, Lecture Notes on Electron Correlation and Magnetism, Series in Modern Condensed Matter Physics (World Scientific, Singapore, 1999).

[52] P. Rhodes and E. P. Wohlfarth, The effective Curie-Weiss constant of ferromagnetic metals and alloys, Proc. R. Soc. A 273, 247 (1963).

[53] J.E. Hirsch, Metallic ferromagnetism in a single-band model. II. Finite-temperature magnetic properties, Phys. Rev. B 40, 9061 (1989).

[54] C. Aron and G. Kotliar, Analytic theory of Hund's metals: A renormalization group perspective, Phys. Rev. B 91, 041110(R) (2015).

[55] L. de Medici, S. R. Hassan, M. Capone, and X. Dai, OrbitalSelective Mott Transition out of Band Degeneracy Lifting, Phys. Rev. Lett. 102, 126401 (2009).

[56] V. I. Anisimov, I. A. Nekrasov, D. E. Kondakov, T. M. Rice, and M. Sigrist, Orbital-selective Mott-insulator transition in $\mathrm{Ca}_{2-x} \mathrm{Sr}_{x} \mathrm{RuO}_{4}$, Eur. Phys. J. B 25, 191 (2002).
[57] A. I. Lichtenstein, M. I. Katsnelson, and G. Kotliar, FiniteTemperature Magnetism of Transition Metals: An Ab Initio Dynamical Mean-Field Theory, Phys. Rev. Lett. 87, 067205 (2001).

[58] N. D. Mermin and H. Wagner, Absence of Ferromagnetism or Antiferromagnetism in One- or Two-Dimensional Isotropic Heisenberg Models, Phys. Rev. Lett. 17, 1133 (1966).

[59] T. A. Zaleski and T. K. Kopeć, Néel order in the Hubbard model within a spin-charge rotating reference frame approach: Crossover from weak to strong coupling, Phys. Rev. B 77, 125120 (2008).

[60] L. M. Roth, Simple narrow-band model of ferromagnetism due to intra-atomic exchange, Phys. Rev. 149, 306 (1966).

[61] K. I. Kugel and D. I. Khomskii, Crystal structure and magnetic properties of substances with orbital degeneracy, Zh. Eksp. Teor. Fiz. 64, 1429 (1973).

[62] M. Cyrot and C. Lyon-Caen, Orbital superlattice in the degenerate Hubbard model, J. Phys. II (France) 36, 253 (1975).

[63] A. M. Oleś, Antiferromagnetism and correlation of electrons in transition metals, Phys. Rev. B 28, 327 (1983).

[64] A. W. Sandvik and J. Kurkijärvi, Quantum Monte Carlo simulation method for spin systems, Phys. Rev. B 43, 5950 (1991).

[65] N. Kawashima and K. Harada, Recent developments of world-line Monte Carlo methods, J. Phys. Soc. Jpn. 73, 1379 (2004).

[66] B. B. Beard and U.-J. Wiese, Simulations of Discrete Quantum Systems in Continuous Euclidean Time, Phys. Rev. Lett. 77, 5130 (1996).

[67] P. Sengupta, A. W. Sandvik, and D. K. Campbell, Bondorder-wave phase and quantum phase transitions in the one-dimensional extended Hubbard model, Phys. Rev. B 65, 155113 (2002).

[68] O. F. Syljuåsen and A. W. Sandvik, Quantum Monte Carlo with directed loops, Phys. Rev. E 66, 046701 (2002).

[69] M. L. Foo, Y. Wang, S. Watauchi, H. W. Zandbergen, T. He, R. J. Cava, and N.P. Ong, Charge Ordering, Commensurability, and Metallicity in the Phase Diagram of the Layered $\mathrm{Na}_{x} \mathrm{CoO}_{2}$, Phys. Rev. Lett. 92, 247001 (2004).

[70] J. Merino, B. J. Powell, and R. H. McKenzie, Ferromagnetism, paramagnetism, and a Curie-Weiss metal in an electron-doped Hubbard model on a triangular lattice, Phys. Rev. B 73, 235107 (2006).

[71] G. A. Fiete, Colloquium: The spin-incoherent Luttinger liquid, Rev. Mod. Phys. 79, 801 (2007).

[72] D. P. Arovas and A. Auerbach, Functional integral theories of low-dimensional quantum Heisenberg models, Phys. Rev. B 38, 316 (1988).

[73] M. Takahashi, Classical Heisenberg ferromagnet in two dimensions, Phys. Rev. B 36, 3791 (1987).

[74] A. Isacsson and S. M. Girvin, Multiflavor bosonic Hubbard models in the first excited Bloch band of an optical lattice, Phys. Rev. A 72, 053604 (2005).

[75] W. V. Liu and C. Wu, Atomic matter of nonzero-momentum Bose-Einstein condensation and orbital current order, Phys. Rev. A 74, 013607 (2006). 
[76] L. Wang, X. Dai, S. Chen, and X. C. Xie, Magnetism of cold fermionic atoms on the $p$ band of an optical lattice, Phys. Rev. A 78, 023603 (2008).

[77] L. Li, C. Richter, J. Mannhart, and R. C. Ashoori, Coexistence of magnetic order and two-dimensional superconductivity at LaAlO3/SrTiO3 interfaces, Nat. Phys. 7, 762 (2011).

[78] J. A. Bert, B. Kalisky, C. Bell, M. Kim, Y. Hikita, H. Y. Hwang, and K. A. Moler, Direct imaging of the coexistence of ferromagnetism and superconductivity at the LaAlO3/SrTiO3 interface, Nat. Phys. 7, 767 (2011).
[79] K. Michaeli, A. C. Potter, and P. A. Lee, Superconducting and Ferromagnetic Phases in $\mathrm{SrTiO}_{3} / \mathrm{LaAlO}_{3}$ Oxide Interface Structures: Possibility of Finite Momentum Pairing, Phys. Rev. Lett. 108, 117003 (2012).

[80] S. Banerjee, O. Erten, and M. Randeria, Ferromagnetic exchange, spin-orbit coupling and spiral magnetism at the $\mathrm{LaAlO}_{3} / \mathrm{SrTiO}_{3}$ interface, Nat. Phys. 9, 626 (2013).

[81] C. Wu, Orbital Frustration and Ordering in p-Band Mott Insulators, Phys. Rev. Lett. 100, 200406 (2008). 